\title{
Treatment Optimization in Multiple Sclerosis: Canadian MS Working Group Recommendations
}

\author{
Mark S. Freedman, Virginia Devonshire, Pierre Duquette, Paul S. Giacomini, \\ Fabrizio Giuliani, Michael C. Levin, Xavier Montalban, Sarah A. Morrow, \\ Jiwon Oh, Dalia Rotstein, E. Ann Yeh on behalf of the Canadian MS \\ Working Group
}

\begin{abstract}
The Canadian Multiple Sclerosis Working Group has updated its treatment optimization recommendations (TORs) on the optimal use of disease-modifying therapies for patients with all forms of multiple sclerosis (MS). Recommendations provide guidance on initiating effective treatment early in the course of disease, monitoring response to therapy, and modifying or switching therapies to optimize disease control. The current TORs also address the treatment of pediatric MS, progressive MS and the identification and treatment of aggressive forms of the disease. Newer therapies offer improved efficacy, but also have potential safety concerns that must be adequately balanced, notably when treatment sequencing is considered. There are added discussions regarding the management of pregnancy, the future potential of biomarkers and consideration as to when it may be prudent to stop therapy. These TORs are meant to be used and interpreted by all neurologists with a special interest in the management of MS.
\end{abstract}

RÉSUMÉ : L'optimisation des traitements destinés à la sclérose en plaques : les recommandations du Canadian Multiple Sclerosis Working Group. Le Canadian Multiple Sclerosis Working Group (CMSWG) vient de mettre à jour ses recommandations visant à optimiser l'utilisation de médicaments modificateurs de l'évolution de l'état de santé de patients atteints de toutes les formes de sclérose en plaques (SP). Ces recommandations, rappelons-le, fournissent des lignes directrices quant à l'amorce d'un traitement efficace au début de la maladie mais aussi quant à un suivi de la réponse des patients à un traitement et à des modifications à un traitement, voire un nouveau traitement, dans le but d'optimiser le contrôle de la SP. Les recommandations actuelles ont également abordé le traitement des cas de SP affectant les enfants, la SP progressive ainsi que l'identification et le traitement de formes de la maladie davantage foudroyantes. Bien que les traitements plus récents offrent une efficacité accrue, des problèmes potentiels en matière de sécurité doivent aussi être pris en compte de façon adéquate, notamment lorsqu'une alternance de traitements (treatment sequencing) est envisagée. À noter que d'autres éléments de discussion et considérations ont été ajoutés par le CMSWG au sujet de la prise en charge des patientes enceintes, du potentiel des biomarqueurs dans le futur et du moment où il peut être prudent de cesser un traitement. Toutes ces recommandations sont destinées à être utilisées et interprétées par tous les neurologues qui nourrissent un intérêt particulier à l'égard de la prise en charge de la SP.

Keywords: MULTIPLE SCLEROSIS, THERAPEUTICS, Recommendations, Treatment optimization, Sequencing

doi:10.1017/cjn.2020.66

Can J Neurol Sci. 2020; 47: 437-455

\section{INTRODUCTION}

Considerable advances have been made in the diagnosis and treatment of multiple sclerosis (MS) since the Canadian Multiple Sclerosis Working Group's (CMSWG) treatment optimization recommendations (TORs) in 2013. ${ }^{1}$ The revised 2017 McDonald criteria have facilitated the earlier accurate diagnosis of $\mathrm{MS} ;{ }^{2}$ a new phenotypic classification of progressive MS (PMS) has been proposed; ${ }^{3}$ research has provided insights on pediatric-onset MS (POMS); an increasingly patient-centric approach has raised awareness about the neuropsychiatric and cognitive impacts of MS on daily functioning; and new biomarkers of disease activity and treatment response are emerging that may guide treatment decision-making in the future.

Of particular importance to treatment optimization is the advent of newer disease-modifying therapies (DMTs), which have made the therapeutic landscape more complex but have also provided clinicians and patients with a wealth of options in managing MS throughout the disease course. The past 6 years

From The University of Ottawa and the Ottawa Hospital Research Institute, Ottawa, Ontario, Canada (MSF); University of British Columbia, Vancouver, British Columbia, Canada (VD); Centre hospitalier de l'Université de Montréal, Montreal, Québec, Canada (PD); Montreal Neurological Institute and Hospital, Montreal, Québec, Canada (PSG); University of Alberta, Edmonton, Alberta, Canada (FG); University of Saskatchewan, Saskatoon, Saskatchewan, Canada (MCL); St. Michael's Hospital, Toronto, Ontario, Canada (XM, JO, DR); London Health Sciences Centre, London, Ontario, Canada (SAM); and The Hospital for Sick Children, Toronto, Ontario, Canada (EAY)

Received November 15, 2019. Final Revisions Submitted February 19, 2020. Date of Acceptance March 29, 2020.

Correspondence to: Mark S. Freedman, HBSc MSc MD CSPQ FANA FAAN FRCPC, Professor of Medicine (Neurology), Ottawa, Ontario, Canada. Email: mfreedman@toh.ca 
have seen the approval of three oral therapies (teriflunomide, dimethyl fumarate, and cladribine) and two monoclonal antibody (MAb) infusion agents (alemtuzumab and ocrelizumab) for relapsing MS (RMS). Moreover, three DMTs have recently been approved in some jurisdictions for PMS: ocrelizumab for primary progressive MS (PPMS), and siponimod and cladribine (in the USA) for active secondary progressive MS (SPMS).

The increasing complexity of treatment decision-making prompted the CMSWG to update its TORs to assist clinicians in their management of MS patients.

The overall objectives of this paper are to review the newer aspects of the diagnosis of MS, discuss the clinical considerations when initiating therapy and planning a course of treatment, update recommendations for determining a suboptimal response, and address safety issues. It is hoped that these recommendations will provide guidance to clinicians on sequencing therapies to optimize treatment response throughout the disease course and inform government and policy-makers about the current needs for accessing medications and health technologies.

\section{Methodology}

The Canadian Network of MS Clinics (CNMSC) invited MSF again to chair a CMSWG and produce an updated version of its 2013 recommendations. ${ }^{1}$ The CNMSC funded this endeavor with contributions from several industry sponsors (see Disclosures) to offset the costs of a meeting coordinator, venue, and travel for members, and to assist the individual groups in compiling their recommendations through teleconferences and webinars. No member received any remuneration or honorarium for participation. The CNMSC solicited members to contact MSF about joining the CMSWG; MSF then compiled a list and selected 10 group leaders, who appointed 2 other individuals to their groups. The objective was not to develop formal clinical practice guidelines, which would have necessitated a different approach and a significant amount of class A, level 1 evidence that is summarily lacking for many of the controversial points discussed here. Rather, the task for each group was to address key issues relevant to treatment optimization in clinical practice in accordance with the evidence base available and an interpreted but expert opinion. Included members had to agree to commit the time and effort needed for literature review and discussion, and for attendance at a 1-day meeting held at the time of the Canadian Neurological Sciences Federation meeting in Montreal in June 2019. The 10 subgroups (and team leaders) were Diagnosis (XM); Treatment initiation - Relapsing MS (FG); Treatment initiation - Progressive MS (MCL); Evaluating treatment response - Relapses and progression (DR); Evaluating treatment response - MRI (JO); Evaluating treatment response Cognition (SAM); Treatment sequencing (PSG); Pediatric MS (EAY); Safety (PD); and Other important issues (VD). Subgroups discussed and finalized their recommendations over a 6-month period ending May 2019, whereupon the results of their deliberations were circulated among all members in preparation for the 1-day discussion and finalization meeting. In June 2019, all CMSWG members were invited to attend a meeting in Montreal, Quebec, to discuss the subgroups' recommendations and arrive at a consensus for each recommendation. Group statements were discussed at length and modified until there was full approval with minimal or no concerns with each statement. This paper summarizes those consensus TORs for MS.

\section{Diagnosis of MS}

Recommendation 1: Use of the 2017 McDonald criteria ${ }^{2}$ is recommended for the diagnosis of RMS and PPMS. McDonald criteria should only be applied to patients presenting clinically with events that are considered highly suspicious for CNS demyelination, after exclusion of reasonable alternative diagnoses.

A rapid and accurate diagnosis is essential to enable earlier initiation of treatment, which has been shown to be associated with better outcomes in patients with their first attack of MS, or "clinically isolated syndrome" (CIS), and RMS. ${ }^{4,5}$ Use of the 2017 McDonald criteria enables more first-attack patients to be diagnosed with RMS at baseline with greater sensitivity but with lower specificity. ${ }^{6}$ A major concern of the International Panel (IP) developing the McDonald criteria was misdiagnosis of MS, most notably in patients with nonspecific neurological signs and atypical findings on magnetic resonance imaging (MRI). ${ }^{2,7}$ For that reason, the IP recommended the use of cerebrospinal fluid (CSF) analysis to help eliminate other conditions that may mimic MS if there are atypical findings or diagnostic uncertainty. The classical finding of CSF-specific oligoclonal banding (OCB), which demonstrates the existence of an inflammatory process within the CNS, is now recognized as being the result of longterm intrathecal stimulation of immunoglobulin (Ig) production and can thus substitute for other requirements in the diagnostic criteria for "dissemination in time".

Accordingly, the CMSWG recommends that, first and foremost, the McDonald criteria only be applied to patients presenting clinically with events that are considered highly suspicious for CNS demyelination, and that MRI or CSF findings should always be interpreted by clinicians with expertise in MS, after exclusion of reasonable alternative diagnoses. An MRI should be obtained promptly, ideally within 1 week of the initial presentation. ${ }^{8}$ Use of a gadolinium $(\mathrm{Gd})$ contrast agent with MRI is recommended in patients presenting with their first clinical attack. This can facilitate an earlier diagnosis of MS by demonstrating new (Gd-enhancing) and older lesions, thus fulfilling the requirements for demonstrating "dissemination in time", and can help to rule out other non-MS conditions. Cortical lesions can be used to show "dissemination in space" but are difficult to identify with conventional MRI sequences used in clinical care.

It should be noted that newer MRI techniques (e.g., susceptibility-weighted imaging), including the "central vein sign"") are emerging that may further improve the specificity of current criteria by enabling the differentiation of white matter lesions that are more likely to be MS-type plaques.

The Magnetic Resonance Imaging in Multiple Sclerosis research network has proposed including paraclinical evidence of optic nerve lesions (MRI, visual evoked potentials, or optical coherence tomography [OCT]) to fulfill criteria for dissemination in space. ${ }^{10}$ Although not currently recommended, ${ }^{2}$ identifying asymptomatic optic nerve involvement in patients with optic neuritis may be relevant as supportive evidence in selected cases. Bilateral optic neuritis and poor recovery from attacks would be more suggestive of a neuromyelitis optica spectrum disorder or myelin oligodendrocyte glycoprotein (MOG) antibody-related disease.

CSF studies are useful to identify atypical findings (e.g., significantly elevated protein concentration, pleocytosis with $>50$ cells $/ \mu \mathrm{l}$, or the presence of neutrophils, eosinophils, and atypical cells) that would suggest an alternative diagnosis. ${ }^{11}$ At 
present, agarose gel electrophoresis with isoelectric focusing and immunoblotting or immunofixation for immunoglobulin-G (IgG) is the most sensitive and accepted method of OCB testing. ${ }^{2}$ The CMSWG recommends that CSF studies be performed only by highly qualified laboratories. Routine testing for aquaporin-4 IgG (AQP4-IgG) or MOG antibodies is not recommended in adult patients with typical MS findings. ${ }^{12}$

At present, the radiologically isolated syndrome (RIS), or the incidental finding of MS-like lesions in the absence of clinical signs or symptoms of MS, is not considered an MS phenotype and treatment is generally not indicated. However, it should be noted that an estimated $50 \%$ of individuals with RIS will subsequently develop MS, including PPMS. ${ }^{13}$ Predictors of a future clinical event include spinal cord lesions, OCB in the CSF, and demographic factors (male sex, age < 37 years); ${ }^{13-15}$ MRI lesions demonstrating the "central vein sign" may also have prognostic value. ${ }^{16}$

The McDonald criteria can also be used to diagnose POMS. Pediatric MS will be addressed in a separate section below.

\section{Treatment Initiation - Relapsing MS}

Recommendation 2: All RMS patients should be encouraged to start treatment with a DMT soon after diagnosis to reduce their risk of disability worsening and to improve long-term outcomes.

The principal objective of treatment is to delay or prevent disability accrual by reducing the frequency of relapses and MRI lesions. Selected RIS cases at high risk of a clinical event may be considered for therapy; ${ }^{17,18}$ this recommendation is controversial and ongoing studies will clarify this issue. With respect to CIS, injectable ${ }^{19-23}$ and oral ${ }^{24,25}$ DMTs have been shown to slow the development of RMS by reducing the chances of having relapses, MRI lesions, or disease progression, an effect that appears to be sustained during long-term follow-up. While new diagnostic criteria now categorize many CIS patients as having MS, treatment benefits have been demonstrated even in contemporary CIS cohorts (i.e., not fulfilling 2017 McDonald criteria at their first attack), ${ }^{26}$ and some of these patients can be offered therapy. The impact of early treatment on longer-term disability outcomes is less certain. ${ }^{27-29}$

All DMTs used for CIS, as well as other DMTs approved as initial therapies, have demonstrated efficacy in the relapsing phase of MS to reduce relapses and MRI lesion development and, in some cases, to slow disease progression, as measured by the Expanded Disability Status Scale (EDSS). ${ }^{30-37}$ Treatment within the first 2 years after diagnosis has been shown to produce sustained benefits compared to delayed treatment. ${ }^{38-40}$ A database analysis by the MSBase Study Group reported a significant reduction in the risk of developing SPMS when an injectable DMT was started within 5 years of disease onset. ${ }^{39}$ The Big MS Data Network, which pools data from MSBase and several European patient registries, found that the optimal time to start DMTs to prevent accumulation of long-term disability was within the first 6 months after disease onset. ${ }^{40}$ The caveat is that these analyses of large, uncontrolled patient populations do not constitute high-quality evidence. ${ }^{41}$

Although the primary evidence for all currently available MS treatments is as first-line therapy, in Canada, the DMTs approved for use as starting treatment (base therapy) are five injectable agents (glatiramer acetate, interferon- $\beta$ - 1 a [three formulations], and interferon- $\beta-1 b$ ) and two oral agents (teriflunomide and dimethyl fumarate). One MAb (ocrelizumab) has recently been approved in Canada for initial therapy in patients with more aggressive presentations.

Few studies have directly compared injectable and oral DMTs. ${ }^{42-44}$ Although a recent network meta-analysis suggested that pegylated interferon- $\beta-1 \mathrm{a}$ and dimethyl fumarate have superior efficacy to other base therapies, ${ }^{45}$ there are insufficient data to demonstrate that one base injectable or oral DMT is superior to another. As a result, the choice of treatment will need to be individualized according to disease activity, severity, and comorbidities. After apprising the patient of all available treatment options, discussion will be needed to ascertain the patient's preferences (e.g., side effect profile, oral vs. injectable), risk tolerance, and other factors that may influence treatment selection.

In addition to the base therapies, there are five DMTs available in Canada considered to be higher efficacy: two oral agents (fingolimod and cladribine) and three MAbs (natalizumab, ocrelizumab, and alemtuzumab). All have demonstrated significant efficacy in phase III trials. ${ }^{35,37,46-48}$ Higher-efficacy agents have been commonly studied as initial therapies but are generally reserved for patients with a poor response or tolerability with a base therapy. Escalating to the use of these agents requires assessment and determination of suboptimal response (see below). Some of these agents should be considered as initial or induction therapies for patients presenting with high disease activity, aggressive or rapidly evolving MS at onset. However, the strategy of treatment initiation with a base therapy, with the view to switching within 6-12 months to one of the higherefficacy DMTs, is the most commonly and conservatively applied one today for most patients.

Recommendation 3: Risk stratification, based on demographic and clinical factors known to be associated with early disease worsening, should be performed for individual patients at first presentation and on an ongoing basis. This will assist clinicians in developing an appropriate treatment plan in consultation with patients and enable prompt optimization of the regimen as required.

Patients at risk of a more aggressive clinical course, worse outcomes, and/or a poorer response to DMTs include males, individuals of non-White ethnicity, and those with high-risk clinical/radiological disease factors (Table 1). ${ }^{49-51}$ There is some evidence to suggest that obesity, smoking, and certain comorbidities (e.g., rheumatoid arthritis, vascular disease, and psychiatric disorders) may be associated with more active disease or worse outcomes, ${ }^{52-54}$ but this requires further study.

Evidence of highly active disease (e.g., frequent relapses and new MRI lesions), extensive CNS involvement (multifocal, lesion number and location, and T2 burden of disease), and/or inadequate recovery/repair (residual impairment, higher baseline EDSS score) or cognitive reserve are prognostic of a worse clinical course and poorer long-term outcomes in CIS and RMS. ${ }^{55,56}$ It has been suggested that demographic factors have a lower impact on prognosis than clinical/radiological factors but may modulate the phenotypic expression of disease activity. ${ }^{57}$ For example, one database analysis found that men were more likely to have pyramidal, brain stem, and cerebellar relapses, which were associated with worse outcomes; women more commonly had sensory or visual symptoms, which showed better recovery; and older patients had poorer recovery from relapses. ${ }^{58}$

Considerable progress has been made in researching the prognostic value of biomarkers of axonal damage and 
Table 1: Demographic and clinical/radiological factors that may be associated with a worse prognosis in MS patients at diagnosis

\begin{tabular}{|c|c|}
\hline Demographic & Clinical/radiological \\
\hline $\begin{array}{l}\text { Age }>40 \text { years } \\
\text { Male sex } \\
\text { Non-White ethnicity } \\
\text { Comorbidity }\end{array}$ & $\begin{array}{l}\text { Relapse frequency } \\
\text { Relapse severity } \\
\text { - }>1 \text { moderate or severe attack } \\
\text { - Steroids/hospitalization required } \\
\text { - Severe effect on activities of daily } \\
\text { living } \\
\text { - }>1 \text { functional system affected } \\
\text { - Severe motor/cerebellar/brain stem } \\
\text { involvement } \\
\text { Relapse recovery } \\
\text { - Incomplete recovery } \\
\text { MRI } \\
\text { - New gadolinium (Gd)-enhancing } \\
\text { T1- or T2-weighted lesions } \\
\text { - T2 lesion volume } \\
\text { - Spinal cord lesion } \\
\text { - Brain atrophy }\end{array}$ \\
\hline
\end{tabular}

neurodegeneration, such as neurofilament-light chain in CSF or serum, glial fibrillary acidic protein in CSF, and retinal nerve fiber layer thickness on OCT. ${ }^{59}$ While the results to date are promising, it is premature to recommend biomarker testing as a guide to clinical decision-making. ${ }^{60,61}$

The initial clinical assessment will include a complete patient history, neurological examination and consideration of prognostic factors. An MRI is recommended. Other paraclinical testing may include OCBs in CSF; examination of the visual system (e.g., evoked potentials, OCT) will help to characterize demyelination and may be useful in diagnosis and prognosis. ${ }^{62-64}$ These measures may help to identify patients at higher risk of early worsening who may be candidates for a more aggressive treatment plan - either initiation with a higher-efficacy agent, or closer monitoring and earlier planned escalation. A patient's risk status may become more apparent in the first few years after diagnosis so vigilance is needed to identify ongoing disease activity that may necessitate a prompt change to a more effective agent.

Recommendation 4. The treatment plan should consider the patient's general health status including comorbidities. Wellness efforts, such as smoking cessation, weight reduction, and regular physical activity, should be encouraged. Vitamin D supplementation (600-4000 IU/day) may provide some added clinical benefit.

The patient's general health status is important to consider during treatment planning. Medical and psychiatric comorbidities are highly prevalent in the MS population ${ }^{65}$ and may be associated with relapses, worsening disability, and poorer quality of life. ${ }^{66-69}$ The presence of comorbid conditions will also be an important consideration during treatment selection and optimization. Clinicians need to work in collaboration with other healthcare providers, including the patient's family physician, to ensure that other medical conditions are being adequately managed. ${ }^{70}$

Patients with MS should be encouraged to adopt a healthier lifestyle as part of the overall disease management strategy. There is some evidence to suggest that smoking cessation, avoidance of obesity, a balanced diet, and regular physical activity may reduce symptoms and improve clinical and radiological outcomes. ${ }^{71-73}$ Vitamin D supplementation has been shown to be beneficial in some but not all studies: ${ }^{74-77}$ a dose of 600-4000 IU/day is recommended as add-on therapy with a DMT. ${ }^{78}$

\section{Treatment Initiation - Primary Progressive MS (PPMS)}

Recommendation 5: Clinicians with experience in treating patients with PMS should offer ocrelizumab to PPMS patients with active disease (relapses and/or MRI activity) provided the benefits outweigh the risks.

The diagnosis of PPMS should be based on the 2017 McDonald criteria of 1 year of disability progression determined prospectively or retrospectively, with two of the following criteria: $\geq 1$ T2 lesions (periventricular, cortical, juxtacortical, and infratentorial); $\geq 2 \mathrm{~T} 2$ lesions in the spinal cord; and presence of CSF-specific OCBs. ${ }^{2}$ MRI lesions may be symptomatic or asymptomatic. $^{2}$ The clinical course should be determined annually using the modifiers "active" with or without progression, and "not active" with or without progression, with activity defined as clinical relapses and/or new or enlarging MRI lesions. ${ }^{3}$ Some DMTs have demonstrated modest effects on progression, most notably in younger PPMS patients ( $<51$ years) with ongoing overt inflammatory activity. ${ }^{79,80}$ It is unclear if these agents influence long-standing progression or modify the pathophysiology of progression unrelated to focal inflammation, which may include progression as a result of normal aging.

At present, ocrelizumab is the only approved DMT that has been shown to be effective in PPMS. The agent should be offered to PPMS patients with active disease (relapses and/or MRI activity) based on the results of a phase III study, which reported a relative $25 \%$ reduction in 6-month confirmed disability progression (CDP) versus placebo (i.e.,29.6\% vs. 35.7\%, respectively). ${ }^{79}$ Patients without active inflammatory changes but worsening disability progression may also be candidates for treatment. Early intervention is recommended since it is likely that the benefits of therapy are greatest in the first few years of disease onset and treatment will be less effective later in the disease course. Ocrelizumab is also recommended in PPMS patients with active disease with upper limb impairment. ${ }^{81}$ Treatment can be considered regardless of the patient's age, duration of PPMS, pyramidal functional score, or EDSS score, although younger ( $<51$ years), more recently diagnosed patients with active inflammatory disease are more likely to have a better response. ${ }^{79}$ Caution is recommended when considering treatment for PPMS subgroups that are less likely to benefit from treatment, such as older patients, those with long-standing stable disease (not active, without progression) and/or significant neurological deficits, since the limited benefits may not justify the risks associated with treatment.

Rituximab, an anti-CD20 MAb, may be considered as an alternative therapy for PPMS in regions that permit off-label use in MS due to cost or other considerations. This recommendation is based on a post hoc analysis of a phase II/III trial that showed a significant reduction in time to 3-month CDP in the subgroup of patients aged $<51$ years and/or with Gd-enhancing lesions; ${ }^{80}$ results should be viewed with caution since the overall trial results were negative. (Treatment initiation in SPMS will be discussed below.) 


\section{Evaluating Treatment Response}

Treatment optimization is founded on the principles of prompt recognition of an inadequate response to a given DMT in an individual patient, either due to lack of efficacy or poor tolerability, and a switch to a more effective therapy when required. Unfortunately, there is a lack of consensus on how to define an adequate treatment response. Several CSF or serum biomarkers of disease activity and treatment response have been proposed but have not been sufficiently validated for use in clinical practice.

In consequence, clinicians must infer a treatment response according to the level of disease activity, as demonstrated by relapses and/or active MRI lesions, after a treatment has been initiated. The rationale for this approach is the finding that ongoing relapses and active lesions over the short term are predictive of disability worsening in patients treated with interferon- $\beta$, teriflunomide, and fingolimod. ${ }^{82-85}$ Attaining no evidence of disease activity (NEDA), defined as no relapses, no EDSS worsening, and no MRI lesion activity, is an ideal goal but is difficult to achieve in practice. Most patients will not achieve NEDA even with a high-efficacy DMT, ${ }^{86-90}$ and the NEDA rate will invariably decline during the course of treatment. ${ }^{91}$ NEDA has been criticized as not reflecting the underlying pathophysiology of MS, ${ }^{92}$ and the predictive value of NEDA for long-term disability outcomes has been questioned. ${ }^{93,94}$ Thus, patients and clinicians will need to tolerate some minimal evidence of disease activity (MEDA), a term that has not been adequately defined. In studies to date, minimal evidence of MRI activity $(<3$ new T2 or $<2 \mathrm{Gd}+$ lesions) was not predictive of EDSS worsening over a 6.7-year follow-up. ${ }^{95}$ However, a retrospective analysis reported that the long-term risk of disability $(\mathrm{EDSS} \geq 6$ ) was similar with NEDA and MEDA, when MEDA was defined as no relapses, $<3$ new T2 lesions, and no Gd+ lesions in the first year of treatment. ${ }^{96}$ Additional studies using different definitions of MEDA are required.

Consequently, there is a need to better define what sort of ongoing disease activity indicative of a suboptimal response is of greater clinical concern. The following summarizes the different domains considered by the CMSWG and recommendations as to when a treatment regimen needs to be optimized.

\section{Relapses}

Recommendation 6: The therapeutic response should be evaluated early in RMS patients to determine the benefit of therapy within the first 2 years after DMT initiation. Treatment response should also be continuously evaluated at regular intervals thereafter. Efficacy assessments should be based on at least two time points using clinical and radiological outcomes. It is recommended that treatment be switched in the first 2 years when there is clear evidence of a suboptimal response.

A relapse is defined according to 2017 McDonald criteria: a clinical episode with patient-reported symptoms and objective findings typical of MS reflecting a focal or multifocal inflammatory demyelinating event in the CNS, developing acutely or subacutely, with a duration $\geq 24$ hours, with or without recovery, and in the absence of fever or infection. ${ }^{2}$ In unclear cases of new or recurrent neurologic symptoms sustained $<24$ hours (e.g., Lhermitte's symptom, trigeminal neuralgia and cognitive impairment), a relapse may be confirmed by $\geq 1$ new localizing MRI lesions.
Table 2: Recommended criteria for switching therapies in RRMS. A change in DMT is indicated for patients who meet any of the Major criteria

\begin{tabular}{|c|c|c|}
\hline & Minor & Major \\
\hline Relapse rate & $\begin{array}{l}\text { One relapse in first } 2 \text { years of } \\
\text { treatment }\end{array}$ & $\begin{array}{l}\geq 2 \text { relapses in first year of } \\
\text { treatment }\end{array}$ \\
\hline Severity & $\begin{array}{l}\text { - Mild } \\
\text { - No functional impairment } \\
\text { (school, work, daily } \\
\text { activities, etc.) } \\
\text { - No motor/cerebellar/brain } \\
\text { stem/ sphincter involvement }\end{array}$ & $\begin{array}{l}\text { - Moderate to severe } \\
\text { - Functional impairment } \\
\text { - Motor/cerebellar/brain } \\
\text { stem/sphincter involvement }\end{array}$ \\
\hline Recovery & $\begin{array}{l}\text { - Full recovery at } 6 \text { months } \\
\text { - No functional impairment } \\
\text { - EDSS change } \leq 1 \text { point at } \\
6 \text { months }\end{array}$ & $\begin{array}{l}\text { - Incomplete recovery } \\
\text { - Functional impairment } \\
\text { - EDSS change }>1 \text { point at } \\
6 \text { months }\end{array}$ \\
\hline MRI & - One new lesion & $\begin{array}{l}\text { - } \geq 3 \text { new lesions during } \\
\text { treatment }\end{array}$ \\
\hline
\end{tabular}

$*>1.5$ points if baseline EDSS 0. If baseline EDSS $>5.5$, any EDSS change would be a Major concern.

**>1 spinal cord lesions may warrant a Major concern.

A number of analyses have shown that frequent relapses in the first 2 years after diagnosis are prognostic of poorer disability outcomes during long-term follow-up. ${ }^{95-98}$ On-treatment "breakthrough" relapses appear to be more predictive of worse outcomes than relapses in untreated patients. ${ }^{99}$ Even minimal ongoing disease activity in the 1-2 years after treatment initiation is highly predictive of disability worsening.

The clinical assessment should consider relapse severity and recovery, both of which have been associated with subsequent severe events and poorer outcomes in some but not all studies. ${ }^{100,101}$ This will require a clinical evaluation, if possible during an attack as well as 3-6 months post-attack, to ascertain the degree of recovery. It may be useful to assess the impact of relapses on work/school attendance and daily functioning as an indicator of relapse severity. The phenotype of on-treatment relapses should also be evaluated: relapses affecting bowel/ bladder, cerebellar, pyramidal, and brain stem function have been reported to have the greatest impact on disability accrual. ${ }^{102}$

Accordingly, on-treatment relapses may be stratified as being of Major or Minor concern (Table 2). Minor criteria generally cooccur (e.g., one mild relapse with one new lesion and no functional limitation); a change in therapy may be considered if a Minor criterion (e.g., one mild relapse) is associated with a second worrisome sign (e.g., two new lesions or a lesion of significant size). Patients meeting any of the Major criteria should be considered to have a suboptimal response, and an immediate change in treatment is recommended. It is important to note that evaluation of on-treatment relapses should only be performed once the drug has achieved a full clinical effect; the time to clinical effect is typically 2-6 months after drug initiation and will differ depending on the DMT used (Table 3). Relapses that occur before the maximal efficacy of the drug has been reached should be given less weight.

The same criteria apply for switching therapies in years 2-10 of treatment although the evidence that on-treatment relapses are 
Table 3: Time to effect of disease-modifying therapies. Treatment response should be evaluated after the full clinical effect has been attained

\begin{tabular}{l|l}
\hline DMT & Time to effect \\
\hline$\bullet$ Natalizumab & $\bullet 1$ month \\
\hline - IFN- $\beta$ formulations & $\bullet 3$ months \\
- Teriflunomide & \\
- Dimethyl fumarate & \\
- Fingolimod & \\
- Ocrelizumab & $\bullet$ 6 months \\
\hline - Glatiramer acetate & $\bullet$ 2-year course required for maximal \\
clinical effect (benefit reported in pivotal \\
trials after Year 2 dosing, with MRI \\
e Cladribine & evaluation for re-baselining at month 18) \\
\hline
\end{tabular}

predictive of disability worsening after $>2$ years on therapy is less robust. Re-dosing may be warranted in patients who have received a 2-year course of treatment with an immune reconstitution therapy (cladribine and alemtuzumab) followed by a period off treatment. In such cases, a return of relapse activity may be attributable to a wearing off of the drug's pharmacodynamic effects or an inadequate overall dose.

\section{Disability}

Recommendation 7: The patient's level of physical disability should be evaluated at least once annually. Some of the most useful measures in practice are the EDSS, the Timed 25-foot Walk (T25FW), the 9-Hole Peg Test (9HPT), and the PatientDetermined Disease Steps (PDDS). Changes in disability should be confirmed at 6 months.

The EDSS is the most commonly used standardized and validated measure of disability worsening. ${ }^{103}$ Scoring is based on a standard neurological examination of seven functional systems (plus Other) as well as ambulation and use of assistive devices. Fully ambulatory patients receive a score $\leq 4.0$; scores of 4.5 to 8.0 are based on the distance walked with or without aid. A change in EDSS score confirmed at 6 months has become the new standard for differentiating relapse-related residual impairment from disability progression. However, it should be noted that confirmation at 6 months may overestimate disability accrual by up to $30 \%$, especially in younger patients. ${ }^{104}$

The T25FW, a component of the Multiple Sclerosis Functional Composite (MSFC), is another validated method, is easy to perform, and is recommended as part of the routine patient assessment. ${ }^{105,106} \mathrm{~A}>20 \%$ change is considered to be clinically meaningful. ${ }^{105,106}$ Assessments should consider factors that may influence ambulatory ability, such as activity prior to the assessment, distance walked to the clinic, ambient temperature, fatigue, depression, cognitive function, and coexisting medical conditions. A second component of the MSFC, the 9HPT, is also useful for evaluating upper limb impairment and manual dexterity, both of which are especially important to MS patients. ${ }^{107} \mathrm{~A}>20 \%$ change in test scores is considered to be clinically meaningful. ${ }^{107}$
There is a need for further development of patient-reported outcomes (PROs) to provide patient-centered and relevant measures of disability severity and progression. At present, one of the few validated PROs for disability is the PDDS, in which patients rate their ambulation from 0 (normal) to 8 (bedridden). ${ }^{108}$ PDDS and EDSS scores have been shown to be strongly correlated, notably for pyramidal and cerebellar functional system scores. ${ }^{109}$ Step count can be reliably estimated based on the average of 2 days in patients who walk unaided. ${ }^{110}$ A change of 800 steps/day corresponds to a one-point worsening in PDDS and is considered to be clinically meaningful. ${ }^{11}$

The previous iteration of the TORs included disability progression as one of the measures for determining the level of concern. ${ }^{1}$ However, residual disability following a relapse more likely represents incomplete relapse recovery rather than true, irreversible disease progression arising from cumulative neurodegeneration and axonal loss. Thus, functional impairment or EDSS change $>1$ point at 6 months would be classified as a Major concern and treatment optimization would be recommended (Table 2). A worsening of EDSS score in the absence of relapses would in principle indicate that the patient is in the SPMS phase (see below), but progression independent of relapse activity has recently been documented to be prevalent in RRMS, blurring the difference between RRMS and SPMS. ${ }^{112}$

\section{MRI}

Recommendation 8. A re-baseline MRI should be obtained after initiating or changing treatment once the DMT is deemed to be fully effective (Table 3). Follow-up MRIs should be obtained annually for the first few years of treatment.

During disease-modifying treatment, brain/cervical spine MRIs are most commonly used to establish a new baseline after treatment initiation, to detect clinically silent breakthrough disease activity, for progressive multifocal leukoencephalopathy (PML) surveillance, and to reevaluate patients with new clinical deterioration. ${ }^{113}$ The optimal timing of an MRI will depend on the time to maximal clinical effect of the DMT (Table 3) and the patient's level of disease activity. ${ }^{113}$ A standardized MRI protocol for brain/cervical spine imaging should be used that meets accepted minimal specifications. Whenever feasible, the same scanner and sequences should be used for sequential MRIs to minimize inter-rater variability in detecting lesions and lesion volume changes. ${ }^{113}$

Contrast enhancement with $\mathrm{Gd}$ is recommended for the initial and re-baseline scans. For the re-baseline scan, the presence of Gd-enhancing lesions may be useful to identify a suboptimal drug response earlier. Gd enhancement is helpful for monitoring clinically silent disease but is not routinely required for follow-up scans. Most enhancing lesions will have a T2 lesion equivalent, T2 lesions are more predictive of outcomes, and the addition of $\mathrm{Gd}$ does not appear to provide much additional information. ${ }^{114-116} \mathrm{Gd}$ may be clinically useful if there is a concern about an alternative diagnosis or when confluent lesions preclude the detection of new lesions on T2-weighted imaging alone. ${ }^{113}$ Caution is now advised about the regular use of $\mathrm{Gd}$, especially in early MS, due to the risk of Gd accumulation in certain areas of the brain with frequent use. ${ }^{117}$

Spinal cord imaging can identify asymptomatic spinal cord lesions that can occur in some patients without clinical symptoms 
or lesions on brain MRI. ${ }^{18,119}$ Periodic spinal cord imaging to identify lesions, even in the absence of new symptoms attributable to the spinal cord, may be useful in selected patients, particularly in those with spinal cord-predominant disease. The recommended sequences include a sagittal T2-weighted and a proton attenuation (STIR) or T1-weighted inversion recovery sequence with phase-sensitive reconstruction. ${ }^{113}$ Axial T2 or T2* and post-contrast (Gd) axial $\mathrm{T} 1$ through the lesions is recommended. ${ }^{113}$ However, routine spinal cord imaging may not be feasible in settings of limited resources.

The measurement of brain or spinal cord atrophy is emerging as an important indicator of disease severity, ${ }^{120}$ and a substantial rate of brain atrophy ( $>0.9-1.2 \%$ /year) may have prognostic value and is clinically worrisome. ${ }^{121,122}$ However, a number of technical issues preclude routine use in clinical practice, including lack of standardization of image acquisition, postacquisition processing requirements, and lack of standardization of interpretation.

MRIs should be obtained annually in the first few years of treatment; the frequency of MRIs thereafter is at the clinician's discretion and will depend on clinical circumstances. More frequent MRIs are warranted when there is concern about highly active disease, during follow-up of patients on higher-efficacy DMTs, to monitor for PML, and to ensure that there is no PML prior to switching therapies. Less frequent MRIs are needed in patients who have remained clinically stable for several years, but it is advised that MRIs should be obtained periodically to guide future management (due to loss of older scans, change in MRI scanners or patient re-location).

Recommendation 9. New/enlarging T2-weighted MRI lesions while on DMT are correlated with new relapses and clinical disability progression over time. A finding of $\geq 3$ new/ enlarging lesions while on a DMT is considered a suboptimal response, and a change in treatment is recommended (Table 2).

MRI is the most sensitive method for detecting ongoing CNS inflammation and provides a good indication of a suboptimal therapeutic response. ${ }^{95,98,99}$ Several studies have reported that $\geq 3$ new/enlarging lesions in the first year of therapy are predictive of worsening EDSS scores during long-term follow-up. ${ }^{95,123}$ Accordingly, $\geq 3$ new lesions during treatment would be a Major criterion (Table 2). However, any evidence of ongoing disease activity in a treated patient is worrisome, and the presence of fewer new/enlarging T2 lesions will still warrant early reassessment, particularly if accompanied by a relapse. T2 lesion load in the first 5 years has been shown to be more strongly correlated with brain atrophy. ${ }^{124}$ The predictive value of lesions on MRI appears to decline later in the disease course as focal inflammatory activity becomes less relevant to clinical outcomes. The number of new asymptomatic spinal cord lesions that warrants a change in treatment is not known. However, even a single new spinal cord lesion may be a Major concern since studies have demonstrated that spinal cord lesions are associated with poorer clinical outcomes. $^{125,126}$

\section{Cognition}

Recommendation 10. Cognition should be tested regularly and as part of an overall assessment of functional change to detect disease activity, relapse recovery or treatment response. The Symbol Digit Modalities Test (SDMT) is the simplest method for screening for cognitive impairment and for identifying changes in cognition over time. An SDMT should be performed at baseline and every 2-3 years. There is insufficient evidence that changing DMTs will improve cognitive outcomes. Accordingly, treatment optimization based on a change in cognitive function alone is not recommended at this time.

Cognitive dysfunction is found in all MS phenotypes, including even RIS and CIS, with the domains of information processing speed, executive function, verbal fluency, and memory being those most commonly affected. ${ }^{127}$ Impaired cognition has a significant impact on patients' daily functioning, employment/ educational status, driving ability, and quality of life. ${ }^{128-130}$

The CMSWG recommends that a baseline cognitive assessment should be performed in all MS patients, in accordance with recommendations from the Consortium of Multiple Sclerosis Centers and the International Multiple Sclerosis Cognition Society. ${ }^{131}$ While annual cognitive testing has been suggested, ${ }^{131}$ less frequent assessments (every 2-3 years) are recommended to minimize a practice effect. ${ }^{132}$ Evaluations should be performed during periods of clinical stability and adjusted for age ${ }^{133}$ to evaluate changes in cognitive function and screen for cognitive impairments. The Brief International Cognitive Assessment in Multiple Sclerosis would ideally be used to screen for cognitive impairment. Otherwise, the recommended tool is the SDMT, which can be rapidly administered ( $<5$ minutes) and has high sensitivity and reliability. ${ }^{134}$ Cognitive changes often accompany relapses and MRI activity, ${ }^{135}$ and additional SDMT testing may be indicated as part of the assessment of relapses or recovery or to evaluate response to treatment. It is important to note that other MS symptoms, including fatigue, mood and anxiety disorders, pain, sleep disorders, and pathological laughing and crying, as well as selected medications (including cannabis) will have an impact on cognition and/or performance on cognitive tests. Once these conditions have been managed, an earlier SDMT assessment may be warranted. $\mathrm{A} \geq 4$-point change in SDMT score over a 2-3 year period is considered clinically meaningful, ${ }^{132}$ especially when it occurs in conjunction with worsening of neurocognitive symptoms, and may indicate a recurrence of inflammatory activity, infection and/or treatment nonadherence. Prompt investigations, including MRI, are recommended. If cognitive impairment is detected and there is no clear explanation (e.g., cannabis use), referral for more extensive neuropsychological testing and discussion of potential contributing factors may be warranted. It is generally useful to have a family member or caregiver present when discussing the benefits and risks of various treatment options in a patient with cognitive impairment.

Several studies have suggested that DMTs may improve cognitive function or slow cognitive decline, ${ }^{136-141}$ although it is unclear if this is due to a reduction in MRI disease seen with these agents. The overall evidence does not link any specific agent consistently with improvement in cognitive function nor does it support switching agents to benefit cognition. Accordingly, although cognitive function is an important aspect of MS management, there is insufficient evidence at this time to support a change in DMT based on cognitive performance alone. However, the choice of treatment may be influenced by cognitive factors, such as the simplicity of adhering to a specific treatment regimen, or the ability to be cognizant of changes suggestive of PML. For example, a therapy with a lower PML risk may be warranted if the patient is unable to reliably contact his/her neurologist with new symptoms suggestive of PML, if the patient 
does not have a caregiver who can reliably monitor for these changes, or if the patient is poorly compliant with monitoring blood work or MRIs.

\section{Treatment Sequencing}

Recommendation 11. Most RMS patients can be expected to require more than one DMT during the treatment course to control their disease and limit worsening disability. Escalation to a higher-efficacy therapy is generally recommended for treated patients who meet a Major criterion (Table 2). When sequencing therapies, clinicians should recognize that a given therapy may have an impact on future treatment choices. Prior to initiating treatment, the clinician should develop a plan as to how medications might be sequenced so that safety concerns or other factors will not limit subsequent treatment options or delay the initiation of the next DMT.

After initiating a DMT, there is a need for close monitoring and early detection of breakthrough disease activity with the view to early escalation to a more effective therapy, if required. Clinicians should recognize the presence of negative prognostic factors (Table 1) and be prepared to act early by switching to a more effective DMT in these higher-risk individuals. A new baseline MRI on treatment is recommended (see Recommendation 8). Clinical response should be evaluated at 6-12 months after DMT initiation since ongoing disease activity is associated with poorer outcomes. The association of early breakthrough disease and disability accrual is strongest in the first 2 years on therapy but remains predictive for 5 years after treatment initiation. ${ }^{97,99}$

Escalation to a higher-efficacy therapy is generally recommended for treated patients who meet a Major criterion (Table 2) indicative of breakthrough disease activity or severity. ${ }^{142}$ A DMT with a different mode of action may be preferred. A lateral switch from one base therapy (injectable or oral) to another may be considered in patients with poor tolerability/adherence. However, the need to switch provides clinicians with an opportunity to escalate therapy to improve efficacy, which may be preferable, especially in patients with negative prognostic factors.

There are few clinical concerns when switching patients from a base therapy to a higher-efficacy DMT. A washout period, that is, a period off treatment to allow the drug to clear and physiologic parameters to normalize, will generally not be required. Blood test results (e.g., lymphocyte count, hepatic enzymes) should be in the normal range before the next treatment is initiated. When discontinuing teriflunomide, an active elimination protocol is used to wash out the drug. Vigilance for PML is recommended for patients switching from DMTs with a known risk of PML (dimethyl fumarate, fingolimod, natalizumab, and ocrelizumab). A lumbar puncture may be considered to rule out John Cunningham virus (JCV) in CSF, especially if switching from one of these medications to a potent immunosuppressive drug, such as cladribine or alemtuzumab.

There are no phase III studies to date comparing higherefficacy therapies. Thus, the choice of higher-efficacy agent will depend on the severity of ongoing disease activity, and the relative benefits and risks of the therapy as evaluated by the clinician and patient. Two treatment approaches are available: continuous immunomodulation (natalizumab, fingolimod, and ocrelizumab) and intermittent immunomodulation with immune reconstitution (cladribine and alemtuzumab), with both approaches associated with a somewhat unique risk profile.
Natalizumab, which blocks immune cell trafficking into the CNS, is often employed as a rescue therapy in patients with aggressive MS due to its high efficacy and rapid onset of action. It can generally be used for up to 18 months regardless of JCV antibody status or JCV Ab index. The drug can be maintained thereafter in patients who are JCV Ab-negative, with close monitoring of JCV serostatus every 3-6 months and clinical/MRI vigilance for PML. JCV Ab-positive patients can continue on natalizumab up to 24 months if the JCV Ab index value $\leq 0.9$ but caution is advised if treatment is maintained beyond 24 months. If the regimen is continued, it is important to include in the clinical notes that the patient has acknowledged the increasing risk of PML with cumulative drug exposure and has agreed to close clinical/MRI surveillance. JCV Ab+ patients on natalizumab $>2$ years will generally be switched to another DMT, ideally during a period of stable disease. Extended interval dosing (300 mg q6-8 weeks) with natalizumab has been proposed as a means of possibly reducing PML risk; ${ }^{143}$ PML cases have still been reported with extended interval dosing, ${ }^{144}$ and patients must be fully informed of the risk of PML. All patients will require a screening MRI when discontinuing natalizumab; a lumbar puncture to detect JCV DNA in CSF may be considered. The risk of disease reactivation after natalizumab discontinuation may be mitigated somewhat if treatment is switched during a period of relative disease quiescence or if the transition time is $<$ 1-2 months. ${ }^{145,146}$ There are limited data that switching from natalizumab to alemtuzumab is more effective than to fingolimod. ${ }^{147}$ However, switching from natalizumab to any DMT with a long duration of action may be considered a high-risk approach since treatment could not be withdrawn in such circumstances if the patient had subclinical PML.

Fingolimod, which sequesters lymphocytes in secondary lymphoid organs, may also be considered when there is a need to switch to a higher-efficacy therapy. The principal safety concerns are the risk of opportunistic infections associated with chronic immunosuppression, and clinical worsening on drug discontinuation reported in some but not all studies. ${ }^{148,149}$ There are few data on sequencing from fingolimod to other high-efficacy DMTs. There have been case reports of clinical worsening after switching from fingolimod to ocrelizumab, rituximab, or alemtuzumab. ${ }^{150-152}$ Conversely, there are reports that rituximab and alemtuzumab are effective in patients with an inadequate response to fingolimod, ${ }^{153,154}$ although persistent $\mathrm{T}$ cell depletion may occur after a fingolimod-alemtuzumab switch. ${ }^{155}$

A washout period has traditionally been recommended when switching among second-line agents to allow lymphocyte counts and other indices (e.g., liver enzymes) to return to normal. The caveat is that anti-trafficking drugs, such as fingolimod and natalizumab, are prone to disease "rebound" in the first 3-6 months after cessation, so a washout period $<3$ months is generally advised. It should be noted that when switching from fingolimod to alemtuzumab, lymphocyte counts should return to normal since alemtuzumab may be less effective if initiated when lymphocytes are still sequestered in secondary lymphoid organs.

A preferred approach when sequencing from a base therapy may be to switch to a cell-depleting agent (cladribine, ocrelizumab, and alemtuzumab). Cladribine is an oral drug that is administered for 8-10 days per year for 2 years (cumulative dose $3.5 \mathrm{mg} / \mathrm{kg}$ ) and which produces significant suppression of $\mathrm{T}$ and $\mathrm{B}$ cells. The main safety consideration is moderate-to-severe 
lymphopenia, which may be minimized if treatment is initiated in patients with a normal lymphocyte count (grade 0 lymphopenia at start, grade 1 prior to Year 2 dosing). ${ }^{156}$ There is no reported increase in infections with the exception of herpes zoster. ${ }^{157}$ After the 2-year course of treatment, patients with an adequate response to cladribine who subsequently experience breakthrough disease may be re-dosed with cladribine or switched to another DMT. ${ }^{158}$ There are no data on the optimal strategy nor on the safety and efficacy of sequencing cladribine with other agents.

Ocrelizumab is an anti-CD20 MAb that is dosed every 6 months to produce chronic suppression of B cells. Infusionrelated reactions are common but generally mild to moderate in severity. ${ }^{159}$ There is an increased risk of infections (upper respiratory tract, urinary tract, and herpes zoster) but no reported increase in opportunistic infections. ${ }^{37}$ PML has rarely occurred; a small number of cases have been reported after switching from a high-efficacy therapy, and one case has been reported in an older patient who was previously untreated. ${ }^{160}$ There have been case reports of hepatitis B virus reactivation during therapy. ${ }^{161}$ Treatment for up to 6 years has been reported to be effective. ${ }^{162}$ An emerging concern is that chronic B cell suppression with ocrelizumab or rituximab has been associated with cases of hypogammaglobulinemia. ${ }^{163,164}$ There are no published data on the safety or efficacy of sequencing DMTs in patients with an inadequate response to ocrelizumab.

Alemtuzumab is an anti-CD52 MAb administered for 8 treatment days over 2 years, which results in profound $\mathrm{T}$ and $\mathrm{B}$ cell suppression. T cell depletion is sustained for several years, whereas B cells hyper-repopulate within 6-9 months. ${ }^{165}$ About $90 \%$ of patients experience infusion reactions despite pretreatment with corticosteroids, antipyretics, and antihistamines. ${ }^{48}$ Thyroid disorders are very common; other secondary autoimmunities are less common. Prolonged T cell suppression is also associated with a risk of infections, including serious opportunistic infections. ${ }^{166} \mathrm{~A}$ further concern is the occurrence of ischemic or hemorrhagic stroke and cervical arterial dissection, which has necessitated a change to the US product label. ${ }^{167}$ The effectiveness of a 2-year treatment course has been demonstrated for up to 9 years. ${ }^{168}$ Patients who experience a return of disease activity may benefit from a third course of treatment. ${ }^{169}$ There are limited data on switching patients to another DMT. B cell-directed therapy has been proposed for patients with B cell-mediated disease activation or autoimmunity associated with alemtuzumab treatment, ${ }^{170-172}$ but additional data are required.

Autologous hematopoietic stem cell transplantation (AHSCT) may be considered relatively early in the treatment course for younger patients $\left(18-31\right.$ years $^{173}$ ) with rapidly evolving MS. Preliminary data suggest that in patients with an inadequate response to a DMT, AHSCT may be more effective than a switch to another DMT, ${ }^{174}$ although it should be noted that in that study, some higher-efficacy agents (ocrelizumab and alemtuzumab) were not included. AHSCT may also be considered in patients with early aggressive disease with a poor prognosis or worsening disability despite treatment with a higher-efficacy DMT. $^{175}$

\section{De-escalation and discontinuation in RMS}

There are limited data on the long-term safety of chronic immunosuppression. De-escalation may be considered in patients on chronic immunosuppressant therapy ( $>5$ years) who have been clinically stable for $>5$ years. It should be noted that disease stability $>5$ years in younger patients $(<60$ years) is unlikely to indicate treatment success and a maintenance therapy will generally be required. There are limited data on the use of IFN- $\beta$, glatiramer acetate, teriflunomide, and DMF as de-escalation agents following natalizumab. ${ }^{176-179}$ Close monitoring for breakthrough disease activity and/or worsening disability (clinic visits every 6-12 months, annual MRIs) is advised following de-escalation. The higher-efficacy DMT previously used should be re-instituted or re-dosed if there is clinical/radiological evidence of breakthrough disease activity.

Clinicians may consider stopping treatment in RMS patients $>60$ years who have been clinically stable for a prolonged period (5-10 years). ${ }^{180,181}$ Older patients have a poorer response to DMTs, an increasing risk of infections and other adverse effects due to immunosenescence, and a higher burden of comorbidities. ${ }^{182,183}$ Close monitoring is required after drug discontinuation with the view to re-initiating therapy if there is breakthrough disease activity. A caveat is that the clinical consequences of breakthrough disease may be significant, since patients later in the disease course show poorer recovery from relapses.

Recommendation 12. Prior to initiating a DMT and throughout the treatment course, clinicians should adhere to a standard screening and monitoring protocol to minimize treatment-associated risks. Screening should include an assessment of contraindications and comorbidities that may influence treatment choice.

Prior to treatment initiation, a number of procedures are recommended depending on the DMT selected, in accordance with individual product monographs. These may include a complete blood cell count with lymphocytes, liver function testing, blood pressure, and urinalysis. Pregnancy testing is recommended for all women of childbearing age. Patients' immunization status should be evaluated to ensure that vaccinations are up to date; of particular importance is vaccination against varicella in patients without prior exposure to chicken pox.

A comprehensive screening and monitoring protocol is required prior to initiating a higher-efficacy DMT and would ideally be performed earlier in the treatment course. A protocol would include screening for latent or active infections (e.g., tuberculosis, hepatitis B, human papillomavirus). Either the tuberculin skin test or interferon-gamma release assay (IGRA) can be used to screen for latent TB infection. ${ }^{184}$ It should be noted that lymphopenia during IGRA testing may increase the risk of a false-negative result. ${ }^{185}$

Required vaccines should ideally be administered before starting an immune-suppressing DMT; administration of live vaccines during treatment is expressly contraindicated. However, for patients with active disease at higher risk of relapse, it is generally recommended to start the DMT as soon as possible even if immunization must be delayed; closer monitoring for infections in such circumstances would be warranted. Product monographs do not specify when vaccines may be administered after DMT discontinuation. As a general guide, vaccination may be considered once lymphocyte counts have returned to normal range, in accordance with the pharmacodynamics of the specific DMT.

PML risk assessment and strategies to minimize risk are required for DMTs known to be associated with PML (primarily 
natalizumab, but also fingolimod, dimethyl fumarate, and ocrelizumab). A JCV Ab index should be routinely obtained during treatment with natalizumab. The utility of the JCV Ab index for PML risk stratification with other DMTs has not been demonstrated. Index values may be altered by anti-CD20 agents and results should be interpreted with caution. ${ }^{186}$ It is not known if switching from natalizumab to another high-efficacy DMT increases the risk of PML. A concern is the reported occurrence of carryover cases of PML after sequential DMTs. ${ }^{187,188}$ A recent MRI ( $<6$ months) to rule out subclinical PML is recommended prior to switching to/from a higher-efficacy DMT. Vigilance for PML is recommended during the treatment course and for 6-12 months after DMT discontinuation.

It will be necessary to re-evaluate the benefits and risks of therapy as the patient ages. The benefits of treatment may decline as a result of age-related changes in immune function (immunosenescence), whereas the risks will increase due to the development of comorbidities, greater susceptibility to treatment-related adverse effects, and other factors. ${ }^{189}$ Close monitoring for infections is recommended in older patients ( $>50$ years) who continue on treatment.

\section{Treatment of Secondary Progressive MS}

Recommendation 13. Ongoing treatment of patients transitioning to SPMS who still have active inflammatory disease is recommended. Use of siponimod, now approved in Canada, may be considered. Consider stopping treatment in patients with SPMS characterized by progression without inflammatory disease activity, with close monitoring to identify breakthrough inflammatory disease activity.

SPMS is defined as progressive accumulation of disability after an initial relapsing course and may be categorized as active with or without progression, not active with progression, and not active without progression (stable disease). ${ }^{3}$ A careful assessment of relapses is needed since pseudorelapses are very common in PMS.

There are several unknown factors regarding the treatment of SPMS. There are no data on the potential benefits of continuing a higher-efficacy DMT after SPMS onset and it is unclear if the current DMT should be maintained after the transition to SPMS. Clinical trials of higher-efficacy agents (e.g., natalizumab, alemtuzumab, and fingolimod) have demonstrated limited or no benefit in patients with progressive disease. ${ }^{190-192}$

It is generally advised to continue with the current DMT after the onset of SPMS since many patients will have ongoing inflammatory disease; ${ }^{193,194}$ subclinical disease activity may worsen if treatment is withdrawn. A change in treatment may be warranted in patients with active SPMS who continue to have relapses or new MRI lesions, with the caveat that there is insufficient evidence to identify criteria for a suboptimal response in patients with SPMS. Accordingly, the CMSWG recommends that the current DMT should be maintained or treatment should be escalated, in patients who transition to SPMS who continue to have active disease (relapses, new MRI lesions). Treatment may be maintained but should not be initiated in those with stable (no relapses or disability progression) SPMS.

Siponimod, a selective sphingosine 1-phosphate receptor-1,5 modulator, has been approved in Canada and other countries for active SPMS; ${ }^{195}$ approval was based on the results of a phase III trial demonstrating a relative $21 \%$ reduction in 3 -month CDP versus placebo (i.e.,CDP $26 \%$ vs. $32 \%$, respectively). ${ }^{196}$ Use of siponimod, may be considered for the treatment of patients with active SPMS. At present, there are no data on whether patients on another DMT during the transition to SPMS would benefit from a switch to siponimod.

Oral cladribine, a purine analog, has also been approved in some countries (not Canada) for active SPMS, ${ }^{197}$ based on preliminary results from randomized trials of injectable formulations. ${ }^{198,199}$ At present, oral cladribine is generally not recommended for the treatment of non-active PMS. There are no data on whether cladribine-treated patients who transition to SPMS would benefit from continuing on the regimen or switching to siponimod or another high-efficacy DMT.

\section{Treatment discontinuation in PMS}

The CMSWG recommends that consideration be given to discontinuing treatment in inactive patients with progression, especially if older ( $>60$ years) with a prolonged period ( $>5$ years) with no new inflammatory disease activity. Preliminary data suggest that treatment may be discontinued in patients $>60$ years with no evidence of inflammatory activity for $>2$ years. ${ }^{180,181}$ An MSBase analysis found that the time to relapse was similar in SPMS patients who stopped versus continued on interferon- $\beta$, but there was a significantly shorter time to disability worsening. ${ }^{200}$ However, it should be noted that a lack of disease activity may indicate a therapeutic effect and discontinuation may result in increased disease activity. Moreover, maintaining treatment may benefit outcomes other than EDSS, such as inflammatory activity, progression on the MSFC, upper limb function, and patient quality of life. ${ }^{190,201,202}$ The recommendation to discontinue treatment in some cases is not meant to restrict DMT use: individual patients may have clinical features or preferences that would make ongoing DMT use a reasonable option. ${ }^{203}$ Close clinical and MRI monitoring every 6-12 months for the first few years after treatment discontinuation is advised to identify breakthrough disease activity. In PPMS patients, the potential benefits and risks of therapy will change during the course of treatment and will need to be re-evaluated on an ongoing basis, notably in older individuals and patients who no longer manifest active disease.

\section{Safety and Other Considerations}

Recommendation 14. Shared decision-making is important when selecting the optimal treatment for individual patients. The potential benefits of specific DMTs must be weighed against the risk of short- and long-term adverse effects associated with that agent. All patients must be fully informed of the potential risks associated with treatment before a DMT is initiated.

The safety of DMTs derives primarily from data obtained in clinical trials and real-world observations, which provide a clearer picture of potential short-term than of long-term adverse effects. New adverse events do emerge in the post-marketing period, and it is important that clinicians exercise caution with newer agents and report adverse events to regulatory authorities. This is especially important now that many patients will be exposed to more than one DMT during their treatment course, and the risk of cumulative toxicities with sequential therapy is largely unknown. Neurologists will need to become familiar with autoimmune, infectious, and oncologic conditions and be prepared to face still unknown complications. 
All patients must be fully informed of potential adverse effects and possible long-term toxicities associated with treatment. ${ }^{204}$ Clinicians should document that patients have acknowledged potential risks. When switching to a higher-efficacy DMT, they must agree beforehand to adhere to the pretreatment protocol and safety monitoring requirements for that drug.

\section{OTHER IsSues}

Recommendation 15. Female MS patients of childbearing age should use a reliable method of contraception. Discontinuation of DMTs is generally recommended prior to conception.

Clinicians and patients need to develop a pregnancy plan that would enable a period of treatment to stabilize disease while avoiding potentially harmful drug effects on the fetus. A few months of treatment and stabilization of disease in an active patient prior to pregnancy may reduce the risk of postpartum relapse. ${ }^{205}$ Pregnancy would ideally be scheduled for a period when disease activity has been low for a year. ${ }^{206}$ For patients with minimal disease activity, glatiramer acetate and interferon- $\beta$ drugs can be used up until pregnancy with no washout period required. ${ }^{207}$ Treatment can be continued through pregnancy and breastfeeding if the benefits outweigh the risks. A washout may not be required with dimethyl fumarate due to the drug's short half-life. Teriflunomide should be used only in cases where the patient understands and agrees to strict contraception prior to pregnancy and to undergo an active elimination protocol prior to pregnancy; confirmation of drug elimination with a blood test prior to conception is recommended. ${ }^{207}$

For patients with highly active disease who are contemplating pregnancy within 2 years, anti-trafficking drugs (fingolimod and natalizumab) should be avoided due to the risk of worsening MS after drug discontinuation and potential teratogenic effects with fingolimod. ${ }^{208}$ Use of a long-acting cell-depleting therapy (e.g., cladribine, alemtuzumab, and ocrelizumab) would be preferred; patients should wait for 4 months after the last dose of alemtuzumab or 6 months after the last dose of cladribine or ocrelizumab before trying to conceive. ${ }^{207}$ In high-risk patients, use of natalizumab and ocrelizumab can be considered up to conception after extensive discussion with an experienced clinician about the potential benefits and risks. ${ }^{209}$ There are some data to suggest that these agents are not transported across the placenta until the second trimester. Use of natalizumab (q4-8 weeks) can be considered for the first two trimesters in patients with aggressive MS during pregnancy, but hematologic monitoring of the infant after childbirth will be required. ${ }^{210}$ With alemtuzumab, close surveillance of new onset thyroid dysfunction during pregnancy is advised. Cytotoxic agents (e.g., mitoxantrone, cyclophosphamide) should be avoided in women of childbearing age; if disease activity warrants their use, the risk of infertility should be discussed with the patient beforehand.

Relapses during pregnancy should only be treated if they are disabling. The preferred treatment is prednisone, prednisolone, or methylprednisolone for 3-5 days, but these agents should be avoided as much as possible in the first trimester. There is insufficient evidence to support the use of routine steroids or monthly intravenous immunoglobulins (IVIg) during pregnancy. Plasmapheresis can be used in severe, refractory relapses.
Treatment Optimization in Pediatric-Onset MS (POMS)

Recommendation 16. Early treatment of POMS is recommended. All DMTs approved for the adult population have been used in POMS and are likely to be efficacious. Treated children and adolescents with MS should be monitored comprehensively, with standardized monitoring according to the specific DMT.

A diagnosis of POMS should be made in accordance with the 2010 and 2017 McDonald criteria., ${ }^{2,215}$ Risk factors include low serum vitamin D levels, smoking, exposure to second-hand smoke, and obesity. Prepubertal onset is uncommon. A minimum of one T1 hypointense or one T2 hyperintense periventricular lesion on MRI has high specificity for POMS. MOG antibodies are found in one-third of children/ adolescents with an acute demyelinating syndrome ${ }^{216}$ (ADS) and are predictive of a non-MS diagnosis; routine antibody testing is recommended in all ADS cases. Adolescents generally present with more typical adult-like monofocal symptoms. PPMS is extremely rare in pediatric patients, and a progressive disease course would generally suggest an alternative diagnosis. The need for early treatment should be emphasized with patients and caregivers, and a thorough discussion is needed of the goals of therapy, treatment options, potential adverse effects, the patient's risk tolerance, and the importance of adherence to improve outcomes. All DMTs have been used in pediatric patients and efficacy may be similar to what is seen in adult $\mathrm{MS},{ }^{217}$ but only one prospective phase III trial has been published to date. ${ }^{218}$ In that study, fingolimod was superior to interferon- $\beta$ and had a safety profile that was generally similar to what has been seen in adult patients. There was a higher incidence of seizures in the study, so monitoring for seizure-like events is recommended in addition to the standard safety monitoring for fingolimod. If other DMTs are used, the same safety monitoring used for adults should be adapted for pediatric patients. A high proportion of pediatric patients will experience breakthrough relapses on an interferon- $\beta$ or glatiramer acetate, and therefore higherefficacy agents may be considered. However, it should be noted that most patients will have ongoing MRI activity even with a higher-efficacy DMT, such as fingolimod. ${ }^{218,219}$

None of the current DMTs has been shown to influence growth or puberty. However, it is recommended that height, weight, and pubertal stage should be monitored at least every 6 months during treatment. Patients should be counselled to avoid risk factors (smoking and obesity); there are some data suggesting that obesity is associated with a poorer response to interferons. $^{220}$ Vitamin D supplementation (to a maximum of $4000 \mathrm{IU} /$ day) is advised in accordance with MS Society of Canada recommendations. ${ }^{78}$ Regular screening for cognitive impairment is recommended due to the high burden of cognitive dysfunction in this age group and the higher risk of poorer cognitive outcomes later in life. ${ }^{221}$ A further concern is the long-term safety of higher-efficacy DMTs, notably the impact of treatment on hormonal changes and fertility, and the risk of secondary malignancies associated with chronic immunosuppression. Additional data from randomized trials or from patient registries on the safety and efficacy of DMTs in POMS are needed. 
Exclusive breastfeeding for 6 months has been reported to reduce the risk of postpartum relapses. ${ }^{211}$ Clinicians should support exclusive breastfeeding in patients without significant disease activity or fatigue. Breastfeeding should not be discouraged in favor of resuming medication except in cases of highly active disease. Postpartum relapses may be treated with methylprednisolone or IVIg, ${ }^{212,213}$ but resumption of a DMT would be the preferred approach. Glatiramer acetate and interferon- $\beta$ may be used during breastfeeding; fingolimod, teriflunomide, dimethyl fumarate, cladribine, and cytoxic agents should be avoided. ${ }^{214}$

Ocrelizumab or natalizumab should be considered for highly active patients or those with a significant postpartum relapse, since these agents have a more rapid onset of action and extremely low transfer to breast milk. Patients who choose not to breastfeed should be started on a DMT as soon as possible after delivery.

\section{Conclusions}

MS is a chronic, debilitating neurological condition that is associated with a high risk of physical and cognitive impairment. Treatment with a DMT may prevent or delay the onset of worsening disability. Many higher-efficacy options are now available, and much attention has been paid to the risks associated with these therapies. While these concerns are clinically appropriate, they can overshadow the very real risks of undertreating MS or not optimizing therapy throughout the disease course, including the progressive accumulation of disability, poor quality of life, and early mortality among MS patients.

Early treatment is recommended since DMTs appear to provide the most clinical benefit during the inflammatory phase of the disease, when it is more likely that interventions will be able to alter the rate of progression. Patients should be riskstratified at first presentation according to the frequency and severity of symptoms and the extent of disease activity as shown on MRI. Patients with highly aggressive disease are at significant risk of early disability worsening and will require early treatment with a higher-efficacy DMT. Those with more mild-to-moderate disease may start with a base therapy, but always with the plan to escalate therapy as soon as possible if there is breakthrough disease activity that is a concern (Table 2).

This is the essence of treatment optimization: the early recognition that a given treatment is suboptimal - either due to inadequate efficacy in controlling disease activity or poor tolerability - and prompt initiation of a therapy that may be more effective. Treatment optimization generally involves one or more escalations until the disease process is adequately controlled. This can involve continuous immunosuppression with an agent that is administered daily or every few months. It may also be accomplished with an "induction" approach, in which a higher-efficacy treatment is administered for a limited duration, followed by periodic redosing if there is a return of disease activity or de-escalation in the case of stable disease.

Unfortunately, there are no head-to-head data to provide a comparison of the relative benefits and risks of higher-efficacy DMTs, nor is there sufficient evidence to guide sequencing decisions in individual patients. As a result, treatment decisions must necessarily be based on a rational assessment of the individual patient's benefits and risks. Throughout this process, the patient should be fully informed of the goals of therapy, the reasons for intensifying therapy, and the potential adverse effects. Consideration must be given to the patient's preferences to encourage a collaborative approach to therapy, and in recognition that some factors - such as the side effect profile, impact on quality of life, and convenience of taking the drug - will influence the patient's satisfaction with a drug and his/her ability to remain on the regimen.

There remain many "unknowns" about MS treatment: there are uncertainties about the key immune target(s); what influences an individual's response to a given therapy; the best treatment strategy; and the extent to which early, aggressive therapy with the currently available agents will modify the progressive neurodegeneration seen in MS. The hope is that employing the core principles of treatment optimization recognizing an inadequate response early and promptly responding with a more effective treatment - will help to minimize long-term disability, preserve daily functioning, and improve quality of life in our MS patients.

\section{Canadian Multiple Sclerosis Working Group Members}

Douglas L. Arnold, Montreal Neurological Institute and Hospital, Montreal, Québec; Philippe Beauchemin, CHU de Québec-Université Laval, Québec, Québec City; Virender Bhan, Fraser Health MS Clinic, Burnaby, British Columbia; Jodie M. Burton, University of Calgary, Calgary, Alberta; Virginia Devonshire, University of British Columbia, Vancouver, British Columbia; Pierre Duquette, Centre hospitalier de l'Université de Montréal, Montreal, Québec; François Émond, CHU de Québec - Université Laval, Hôpital de l'Enfant-Jésus, Québec, Québec; Mark S. Freedman, The Ottawa Hospital Research Institute, Ottawa, Ontario; Paul S. Giacomini, Montreal Neurological Institute and Hospital, Montreal, Québec; Marc Girard, Centre hospitalier de l'Université de Montréal, Montreal, Québec; Fabrizio Giuliani, University of Alberta, Edmonton, Alberta; Francois Grand'Maison, Clinique de SP Neuro Rive-Sud, Greenfield Park, Québec; Marika Hohol, St. Michael's Hospital, Toronto, Ontario; Olinka Hrebicek, Royal Jubilee Hospital, Victoria, British Columbia; Liesly Lee, Sunnybrook Health Sciences Centre, Toronto, Ontario; Michael C. Levin, University of Saskatchewan, Saskatoon, Saskatchewan; James J. Marriott, University of Manitoba, Winnipeg, Manitoba; Suresh Menon, McMaster University, Hamilton, Ontario; Xavier Montalban, St. Michael's Hospital, Toronto, Ontario; Sarah A. Morrow, London Health Sciences Centre, London, Ontario; Mary Lou Myles, University of Alberta, Edmonton, Alberta; Jiwon Oh, St. Michael's Hospital, Toronto, Ontario; David Patry, Foothills Medical Centre, Calgary, Alberta; Daniela Pohl, Children's Hospital of Eastern Ontario, Ottawa, Ontario; Alexandre Prat, Centre hospitalier de l'Université de Montréal, Montreal, Québec; Dalia Rotstein, St. Michael's Hospital, Toronto, Ontario; Daniel Selchen, St. Michael's Hospital, Toronto, Ontario; Penelope Smyth, University of Alberta Hospital, Edmonton, Alberta; Sunita Venkateswaran, Children's Hospital of Eastern Ontario, Ottawa, Ontario; Reza Vosoughi, St. Michael's Hospital, Toronto, Ontario; and E. Ann Yeh, The Hospital for Sick Children, Toronto, Ontario. 


\section{DisClosures}

The Canadian Treatment Optimization Recommendations was a project of the Canadian MS Working Group (CMSWG) under the aegis of the Canadian Network of MS Clinics. All of the authors have reviewed the final version of this manuscript and have agreed with the recommendations. CMSWG members received no remuneration for their development of the recommendations or the accompanying manuscript. Participants were reimbursed for costs associated with attending the June 2019 meeting. Funding was provided by the Canadian Network of MS Clinics through unrestricted grants obtained from EMD Serono, Roche, Biogen, Sanofi-Genzyme, Teva, and Pendopharm. Corporate donors had no involvement in CMSWG discussions, in the development of the recommendations or in the review of the manuscript. The CMSWG acknowledges the assistance of Anne St-Michel and Steven Manners of Communications Lansdowne for logistics and editorial development, with funding through grants obtained by the Canadian Network of MS Clinics.

\section{Author Disclosures}

Mark S. Freedman has received grants from Sanofi Genzyme, Hoffmann La Roche, and EMD Serono; honoraria from Sanofi Genzyme, Hoffmann La Roche, EMD Serono, Actelion, Janssen/J\&J, Alexion, Biogen, Novartis. and Teva Canada Innovation; and participated in speakers' bureaux for Sanofi Genzyme and EMD Serono.

Virginia Devonshire has received honoraria for attending advisory board meetings for EMD Serono, Roche, Sanofi, and Biogen.

Pierre Duquette has received grants for investigator-initiated trials from Biogen and EMD Serono.

Paul S. Giacomini has received fees for speaking, consulting, and advisory board participation for Novartis, Biogen, EMD Serono, Sanofi Genzyme, Hoffmann La Roche, Teva, Actelion, Alexion, Celgene, and Pendopharm.

Fabrizio Giuliani has received grants from Biogen and Hoffmann La Roche; and honoraria from Novartis, EMD Serono, and Teva.

Michael C. Levin has received honoraria from Biogen, Pendopharm, Hoffmann La Roche, Merck, and Sanofi Genzyme; and non-financial support from Biogen, Pendopharm, and Sanofi Genzyme.

Xavier Montalban has received speaking honoraria and travel expenses from Biogen, Novartis, Sanofi Genzyme, Teva, Hoffmann La Roche, Celgene, Actelion, Excemed, and EMD Serono; has participated in scientific meetings and advisory board meetings for Biogen, Novartis, Sanofi Genzyme, Teva, Hoffmann La Roche, Celgene, Actelion, Excemed, and EMD Serono; and received travel expenses for scientific meeting participation for the MS International Federation and the National MS Society.

Sarah A. Morrow has received grants from the Multiple Sclerosis Society of Canada and CIHR; has acted as site principal investigator for studies with Novartis, Roche, and EMD Serono; and has received research funds for investigator-initiated studies from Hoffmann La Roche, Biogen, Sanofi Genzyme, and EMD Serono.
Jiwon $O h$ has received grants from Biogen, Hoffmann La Roche, and Sanofi Genzyme; and honoraria from Biogen, Hoffmann La Roche, EMD Serono, Celgene, Novartis, and Sanofi Genzyme.

Dalia Rotstein has received research support from the Consortium of MS Centers, the Multiple Sclerosis Society of Canada, and Hoffmann La Roche; and has served as a speaker or consultant for Alexion, Biogen, EMD Serono, Novartis, Hoffmann La Roche, and Sanofi Aventis.

E. Ann Yeh has received grants from Biogen, the National MS Society, the Consortium of MS Centers, the Multiple Sclerosis Society of Canada, the Ontario Institute for Regenerative Medicine, SCN, the Sick Kids Foundation, CBMH, Teva and the Guthy Jackson Foundation; and honoraria from ACI, the US FDA, and Juno.

\section{Statement of Authorship}

MSF designated the subgroups that would develop the preliminary recommendations. The other 10 authors served as team leaders in developing the preliminary recommendations by their subgroups. The 10 subgroups (and team leaders) were Diagnosis (XM); Treatment initiation - Relapsing MS (FG); Treatment initiation - Progressive MS (MCL); Evaluating treatment response - Relapses and progression (DR); Evaluating treatment response - MRI (JO); Evaluating treatment response Cognition (SAM); Treatment sequencing (PSG); Pediatric MS (EAY); Safety (PD); and Other important issues (VD). MSF led the full Working Group discussion to finalize the consensus recommendations. All of the authors (MSF, XM, FG, MCL, DR, JO, SAM, PSG, EAY, PD, and VD) reviewed all drafts and revisions of the manuscript. All of the authors have reviewed the final version of this manuscript and have agreed with the conclusions.

\section{ACKNOWLEDGMENTS}

The CMSWG wishes to thank Drs. Ruth Ann Marrie, University of Manitoba, and Luanne Metz, University of Calgary, for their critical review of the manuscript. Dr F. Émond gratefully acknowledges the work of Dr. Josée Masson-Roy, CHU de Québec, in reviewing the pregnancy and breastfeeding literature.

\section{REFERENCES}

1. Freedman MS, Selchen D, Arnold DL, et al. Treatment optimization in MS: Canadian MS Working Group updated recommendations. Can J Neurol Sci. 2013;40:307-323.

2. Thompson AJ, Banwell BL, Barkhof F, et al. Diagnosis of multiple sclerosis: 2017 revisions of the McDonald criteria. Lancet Neurol. 2018;17:162-173.

3. Lublin FD. New multiple sclerosis phenotypic classification. Eur Neurol. 2014;72 Suppl 1:1-5.

4. Kappos L, Freedman MS, Polman CH, et al. Effect of early versus delayed interferon beta- $1 b$ treatment on disability after a first clinical event suggestive of multiple sclerosis: a 3-year follow-up analysis of the BENEFIT study. Lancet. 2007;370:389-397.

5. Freedman MS, Comi G, De Stefano N, et al. Moving toward earlier treatment of multiple sclerosis: findings from a decade of clinical trials and implications for clinical practice. Mult Scler Relat Disord. 2014;3:147-155.

6. van der Vuurst de Vries RM, Mescheriakova JY, Wong YYM, et al. Application of the 2017 revised McDonald Criteria for multiple 
sclerosis to patients with a typical clinically isolated syndrome. JAMA Neurol. 2018;75:1392-1398.

7. Calabrese M, Gasperini C, Tortorella C, et al. "Better explanations" in multiple sclerosis diagnostic workup: a 3-year longitudinal study. Neurology. 2019;92:e2527-e2537.

8. Traboulsee A, Létourneau-Guillon L, Freedman MS, et al. Canadian Expert Panel Recommendations for MRI use in MS diagnosis and monitoring. Can J Neurol Sci. 2015;42:159-167.

9. Sati P, Oh J, Constable RT, et al. The central vein sign and its clinical evaluation for the diagnosis of multiple sclerosis: a consensus statement from the North American Imaging in Multiple Sclerosis Cooperative. Nat Rev Neurol. 2016;12:714-722.

10. Filippi M, Preziosa P, Meani A, et al. Prediction of a multiple sclerosis diagnosis in patients with clinically isolated syndrome using the 2016 MAGNIMS and 2010 McDonald criteria: a retrospective study. Lancet Neurol. 2018;17:133-142.

11. Stangel M, Fredrikson S, Meinl E, Petzold A, Stüve O, Tumani H. The utility of cerebrospinal fluid analysis in patients with multiple sclerosis. Nat Rev Neurol. 2013;9:267-276.

12. Jarius S, Paul F, Aktas O, et al. MOG encephalomyelitis: international recommendations on diagnosis and antibody testing. J Neuroinflammation. 2018;15:134.

13. Lebrun-Frenay C, Kantarci O, Siva A, et al. Radiologically isolated syndrome: a 10-year follow-up study to identify factors predicting a clinical event (abstract 97). Presented at the 35th Congress of the European Committee for Treatment and Research in Multiple Sclerosis, Stockholm, Sweden, October 11-13, 2019.

14. Okuda DT, Siva A, Kantarci O, et al. Radiologically isolated syndrome: 5-year risk for an initial clinical event. PLoS One. 2014;9:e90509.

15. Makhani N, Lebrun C, Siva A, et al. Oligoclonal bands increase the specificity of MRI criteria to predict multiple sclerosis in children with radiologically isolated syndrome. Mult Scler J Exp Transl Clin. 2019;5:2055217319836664.

16. Suthiphosuwan $\mathrm{S}$, Sati $\mathrm{P}$, Guenette $\mathrm{M}$, et al. The central vein sign in radiologically isolated syndrome. AJNR Am J Neuroradiol. 2019:40:776-783.

17. Tornatore C, Phillips JT, Khan O, Miller AE, Hughes M. Consensus opinion of US neurologists on practice patterns in RIS, CIS, and RRMS: evolution of treatment practices. Neurol Clin Pract. 2016; 6:329-338

18. Alshamrani F, Alnajashi H, Freedman M. Radiologically isolated syndrome: watchful waiting vs. active treatment. Expert Rev Neurother. 2017;17:441-447.

19. Jacobs LD, Beck RW, Simon JH, et al. Intramuscular interferon beta-1a therapy initiated during a first demyelinating event in multiple sclerosis. CHAMPS Study Group. N Engl J Med. 2000; 343:898-904.

20. Comi G, Filippi M, Barkhof F, et al. Effect of early interferon treatment on conversion to definite multiple sclerosis: a randomised study. Lancet. 2001;357:1576-1582.

21. Kappos L, Polman CH, Freedman MS, et al. Treatment with interferon beta- $1 \mathrm{~b}$ delays conversion to clinically definite and McDonald MS in patients with clinically isolated syndromes. Neurology. 2006;67:1242-1249.

22. Comi G, Martinelli V, Rodegher M, et al. Effect of glatiramer acetate on conversion to clinically definite multiple sclerosis in patients with clinically isolated syndrome (PreCISe study): a randomised, double-blind, placebo-controlled trial. Lancet. 2009:374:1503-1511.

23. Comi G, De Stefano N, Freedman MS, et al. Comparison of two dosing frequencies of subcutaneous interferon beta-1a in patients with a first clinical demyelinating event suggestive of multiple sclerosis (REFLEX): a phase 3 randomised controlled trial. Lancet Neurol. 2012;11:33-41.

24. Miller AE, Wolinsky JS, Kappos L, et al. Oral teriflunomide for patients with a first clinical episode suggestive of multiple sclerosis (TOPIC): a randomised, double-blind, placebocontrolled, phase 3 trial. Lancet Neurology. 2014;13:977-986.

25. Leist TP, Comi G, Cree BA, et al. Effect of oral cladribine on time to conversion to clinically definite multiple sclerosis in patients with a first demyelinating event (ORACLE MS): a phase 3 randomised trial. Lancet Neurology. 2014;13:257-267.
26. Freedman MS, Leist TP, Comi G, et al. The efficacy of cladribine tablets in CIS patients retrospectively assigned the diagnosis of MS using modern criteria: results from the ORACLE-MS study. Mult Scler J Exp Transl Clin. 2017;3:2055 217317732802

27. Kinkel RP, Dontchev M, Kollman C, et al. Association between immediate initiation of intramuscular interferon beta-1a at the time of a clinically isolated syndrome and long-term outcomes: a 10-year follow-up of the Controlled High-Risk Avonex Multiple Sclerosis Prevention Study in Ongoing Neurological Surveillance. Arch Neurol. 2012;69:183-190.

28. Kappos L, Edan G, Freedman MS, et al. The 11-year long-term follow-up study from the randomized BENEFIT CIS trial. Neurology. 2016;87:978-987.

29. Miller AE, Vermersch P, Kappos L, et al. Long-term outcomes with teriflunomide in patients with clinically isolated syndrome: results of the TOPIC extension study. Mult Scler Relat Disord. 2019;33:131-138.

30. The IFNB Multiple Sclerosis Study Group. Interferon beta- $1 \mathrm{~b}$ is effective in relapsing-remitting multiple sclerosis. I. Clinical results of a multicenter, randomized, double-blind, placebocontrolled trial. Neurology. 1993;43:655-661.

31. Johnson KP, Brooks BR, Cohen JA, et al. Copolymer 1 reduces relapse rate and improves disability in relapsing-remitting multiple sclerosis: results of a phase III multicenter, double-blind placebo-controlled trial. Neurology. 1995;45:1268-1276.

32. Jacobs LD, Cookfair DL, Rudick RA, et al. Intramuscular interferon beta-1a for disease progression in relapsing multiple sclerosis. Ann Neurol. 1996;39:285-294.

33. PRISMS (Prevention of Relapses and Disability by Interferon beta-1a Subcutaneously in Multiple Sclerosis) Study Group. Randomised double-blind placebo-controlled study of interferon beta-1a in relapsing/remitting multiple sclerosis. Lancet. 1998; 352:1498-1504.

34. O'Connor P, Wolinsky JS, Confavreux C, et al. Randomized trial of oral teriflunomide for relapsing multiple sclerosis. N Engl J Med. 2011;365:1293-1303.

35. Giovannoni G, Comi G, Cook S, et al. A placebo-controlled trial of oral cladribine for relapsing multiple sclerosis. N Engl J Med. 2010; 362:416-426.

36. Gold R, Kappos L, Arnold DL, et al. Placebo-controlled phase 3 study of oral BG-12 for relapsing multiple sclerosis. N Engl J Med. 2012;367:1098-1107.

37. Hauser SL, Bar-Or A, Comi G, et al. Ocrelizumab versus interferon beta-1a in relapsing multiple sclerosis. N Engl J Med. 2017; 376:221-234.

38. Chalmer TA, Baggesen LM, Nørgaard M, et al. Early versus later treatment start in multiple sclerosis: a register-based cohort study. Eur J Neurol. 2018;25:1262-e110.

39. Brown JWL, Coles A, Horakova D, et al. Association of initial disease-modifying therapy with later conversion to secondary progressive multiple sclerosis. JAMA. 2019;321:175-187.

40. Iaffaldano $P$. The optimal time to start treatment in relapsing remitting multiple sclerosis patients: results from the Big Multiple Sclerosis Data Network (abstract 204). Presented at the 34th Congress of the European Committee for Treatment and Research in Multiple Sclerosis, Berlin, Germany, October 10-12, 2018.

41. Gliklich R, Dreyer N, Leavy M, eds. Registries for evaluating patient outcomes: a user's guide. Third edition. Agency for Healthcare Research and Quality publication No. 13(14)-EHC111. Rockville, MD, 2014. www.effectivehealthcare.ahrq.gov/registries-guide-3.cfm. Accessed October 1, 2019.

42. Mikol DD, Barkhof F, Chang P, et al. Comparison of subcutaneous interferon beta-1a with glatiramer acetate in patients with relapsing multiple sclerosis (the REbif vs Glatiramer Acetate in Relapsing MS Disease [REGARD] study): a multicentre, randomised, parallel, open-label trial. Lancet Neurol. 2008;7: 903-914.

43. Fox RJ, Miller DH, Phillips JT, et al. Placebo-controlled phase 3 study of oral BG-12 or glatiramer in multiple sclerosis. N Engl J Med. 2012;367:1087-1097.

44. Vermersch P, Czlonkowska A, Grimaldi LM, et al. Teriflunomide versus subcutaneous interferon beta-1a in patients with relapsing 
multiple sclerosis: a randomised, controlled phase 3 trial. Mult Scler. 2014;20:705-716.

45. Li H, Hu F, Zhang Y, Li K. Comparative efficacy and acceptability of disease-modifying therapies in patients with relapsingremitting multiple sclerosis: a systematic review and network meta-analysis. J Neurol. 2019; epublished May 25, 2019.

46. Polman $\mathrm{CH}$, O'Connor PW, Havrdova E, et al. A randomized, placebo-controlled trial of natalizumab for relapsing multiple sclerosis. N Engl J Med. 2006;354:899-910.

47. Kappos L, Radue EW, O'Connor P, et al. A placebo-controlled trial of oral fingolimod in relapsing multiple sclerosis. N Engl J Med. 2010;362:387-401.

48. Cohen JA, Coles AJ, Arnold DL, et al. Alemtuzumab versus interferon beta $1 \mathrm{a}$ as first-line treatment for patients with relapsing-remitting multiple sclerosis: a randomised controlled phase 3 trial. Lancet. 2012;380:1819-1828.

49. Damasceno A, Von Glehn F, Brandão CO, Damasceno BP, Cendes F. Prognostic indicators for long-term disability in multiple sclerosis patients. J Neurol Sci. 2013;324:29-33.

50. Saeedi J, Rieckmann P, Yee I, Tremlett H. UBC MS clinic neurologists. Characteristics of multiple sclerosis in aboriginals living in British Columbia, Canada. Mult Scler J. 2012;18:1239-1243.

51. Fisniku LK, Brex PA, Altmann DR, et al. Disability and T2 MRI lesions: a 20-year follow-up of patients with relapse onset of multiple sclerosis. Brain. 2008;131(Pt 3):808-817.

52. Manouchehrinia A, Hedström AK, Alfredsson L, Olsson T, Hillert J, Ramanujam R. Association of pre-disease body mass index with multiple sclerosis prognosis. Front Neurol. 2018; 9:232.

53. Tettey P, Siejka D, Simpson S Jr, et al. Frequency of comorbidities and their association with clinical disability and relapse in multiple sclerosis. Neuroepidemiology. 2016;46:106-113.

54. McKay KA, Tremlett H, Fisk JD, et al. Psychiatric comorbidity is associated with disability progression in multiple sclerosis. Neurology. 2018;90:e1316-e1323.

55. Tomassini V, Fanelli F, Prosperini L, Cerqua R, Cavalla P, Pozzilli C. Predicting the profile of increasing disability in multiple sclerosis. Mult Scler. 2019;25:1306-1315.

56. Spelman T, Meyniel C, Rojas JI, et al. Quantifying risk of early relapse in patients with first demyelinating events: prediction in clinical practice. Mult Scler. 2017;23:1346-1357.

57. Tintore M, Rovira À, Río J, et al. Defining high, medium and low impact prognostic factors for developing multiple sclerosis. Brain. 2015;138(Pt 7):1863-1874.

58. Kalincik T, Buzzard K, Jokubaitis V, et al. Risk of relapse phenotype recurrence in multiple sclerosis. Mult Scler. 2014;20:1511-1522.

59. Novakova L, Axelsson M, Malmeström C, et al. Searching for neurodegeneration in multiple sclerosis at clinical onset: diagnostic value of biomarkers. PLoS One. 2018;13:e0194828.

60. Rotstein D, Montalban X. Reaching an evidence-based prognosis for personalized treatment of multiple sclerosis. Nat Rev Neurol. 2019; 15:287-300.

61. Harris VK, Sadiq SA. Disease biomarkers in multiple sclerosis: potential for use in therapeutic decision making. Mol Diagn Ther. 2009;13:225-244.

62. Heidari M, Radcliff AB, McLellan GJ, et al. Evoked potentials as a biomarker of remyelination. Proc Natl Acad Sci USA. 2019; epublished December 16, 2019.

63. Chisari CG, Toro MD, Cimino V, et al. Retinal nerve fiber layer thickness and higher relapse frequency may predict poor recovery after optic neuritis in MS patients. J Clin Med. 2019;8. pii: E2022.

64. Kim NH, Kim HJ, Park CY, Jeong KS. Retinal degeneration after first-ever optic neuritis helps differentiate multiple sclerosis and neuromyelitis optica spectrum disorder. Front Neurol. 2019;10: 1076.

65. Zhang $\mathrm{T}$, Tremlett $\mathrm{H}$, Leung $\mathrm{S}$, et al. Examining the effects of comorbidities on disease-modifying therapy use in multiple sclerosis. Neurology. 2016;86:1287-1295.

66. Kowalec K, McKay KA, Patten SB, et al. Comorbidity increases the risk of relapse in multiple sclerosis: a prospective study. Neurology. 2017;89:2455-2461.
67. Zhang T, Tremlett H, Zhu F, et al. Effects of physical comorbidities on disability progression in multiple sclerosis. Neurology. 2018;90:e419-e427.

68. Scherder R, Kant N, Wolf ET, Pijnenburg B, Scherder EJ. Psychiatric and physical comorbidities and pain in patients with multiple sclerosis. J Pain Res. 2018;11:325-334.

69. Conway DS, Thompson NR, Cohen JA. Influence of hypertension, diabetes, hyperlipidemia, and obstructive lung disease on multiple sclerosis disease course. Mult Scler. 2017;23:277-285.

70. Marrie RA. Comorbidity in multiple sclerosis: implications for patient care. Nat Rev Neurol. 2017;13:375-382.

71. Degelman ML, Herman KM. Smoking and multiple sclerosis: a systematic review and meta-analysis using the Bradford Hill criteria for causation. Mult Scler Relat Disord. 2017;17:207-216.

72. Amato MP, Derfuss T, Hemmer B, et al. Environmental modifiable risk factors for multiple sclerosis: report from the 2016 ECTRIMS focused workshop. Mult Scler. 2017;Jan 6:1352458516686847.

73. Esposito S, Bonavita S, Sparaco M, Gallo A, Tedeschi G. The role of diet in multiple sclerosis: a review. Nutr Neurosci. 2018;21: 377-390.

74. Løken-Amsrud KI, Holmøy T, Bakke SJ, et al. Vitamin D and disease activity in multiple sclerosis before and during interferon- $\beta$ treatment. Neurology. 2012;79:267-273.

75. Fitzgerald KC, Munger KL, Köchert K, et al. Association of vitamin $\mathrm{D}$ levels with multiple sclerosis activity and progression in patients receiving interferon beta-1b. JAMA Neurol. 2015;72: $1458-1465$.

76. Muris AH, Smolders J, Rolf L, et al. Vitamin D status does not affect disability progression of patients with multiple sclerosis over three year follow-up. PLoS One. 2016;11:e0156122.

77. Kampman MT, Steffensen LH, Mellgren SI, Jørgensen L. Effect of vitamin D3 supplementation on relapses, disease progression, and measures of function in persons with multiple sclerosis: exploratory outcomes from a double-blind randomised controlled trial. Mult Scler. 2012;18:1144-51

78. Multiple Sclerosis Society of Canada. Vitamin D and multiple sclerosis recommendations. https://mssociety.ca/library/document/ Vka6RXcnOizNm9sIwuWvroxejlhLqTJ8/original.pdf. Accessed 25 July 2019.

79. Montalban X, Hauser SL, Kappos L, et al. Ocrelizumab versus placebo in primary progressive multiple sclerosis. N Engl J Med. 2017;376:209-220.

80. Hawker K, O'Connor P, Freedman MS, et al. Rituximab in patients with primary progressive multiple sclerosis: results of a randomized double-blind placebo-controlled multicenter trial. Ann Neurol. 2009;66:460-471.

81. Fox EJ, Markowitz C, Applebee A, et al. Ocrelizumab reduces progression of upper extremity impairment in patients with primary progressive multiple sclerosis: findings from the phase III randomized ORATORIO trial. Mult Scler. 2018;24: $1862-1870$.

82. Sormani MP, Li DK, Bruzzi P, et al. Combined MRI lesions and relapses as a surrogate for disability in multiple sclerosis. Neurology. 2011;77:1684-1690.

83. Sormani MP, Gasperini C, Romeo M, et al. Assessing response to interferon- $\beta$ in a multicenter dataset of patients with MS. Neurology. 2016;87:134-140.

84. Sormani MP, Truffinet P, Thangavelu K, Rufi P, Simonson C, De Stefano N. Predicting long-term disability outcomes in patients with MS treated with teriflunomide in TEMSO. Neurol Neuroimmunol Neuroinflamm. 2017;4:e379.

85. Boster A, Hawker K, Ritter S, et al. Disease activity in the first year predicts longer-term clinical outcomes in the pooled population of the phase III FREEDOMS and FREEDOMS II studies (P7.239). Neurology. 2015;84(14 suppl):P7.239.

86. Havrdová E, Galetta S, Hutchinson M, et al. Effect of natalizumab on clinical and radiological disease activity in multiple sclerosis: a retrospective analysis of the Natalizumab Safety and Efficacy in Relapsing-Remitting Multiple Sclerosis (AFFIRM) study. Lancet Neurol. 2009;8:254-260.

87. Gärtner J, Chitnis T, Ghezzi A, et al. Relapse rate and MRI activity in young adult patients with multiple sclerosis: a post hoc analysis 
of phase 3 fingolimod trials. Mult Scler J Exp Transl Clin. 2018; 4:2055217318778610.

88. Giovannoni G, Cook S, Rammohan K, et al. Sustained diseaseactivity-free status in patients with relapsing-remitting multiple sclerosis treated with cladribine tablets in the CLARITY study: a post-hoc and subgroup analysis. Lancet Neurol. 2011;10:329-337.

89. Havrdová E, Arnold DL, Bar-Or A, et al. No evidence of disease activity (NEDA) analysis by epochs in patients with relapsing multiple sclerosis treated with ocrelizumab vs interferon beta-1a. Mult Scler J Exp Transl Clin. 2018;4:2055217318760642.

90. Coles AJ, Twyman CL, Arnold DL, et al. Alemtuzumab for patients with relapsing multiple sclerosis after disease-modifying therapy: a randomised controlled phase 3 trial. Lancet. 2012;380:1829-1839.

91. Rotstein DL, Healy BC, Malik MT, Chitnis T, Weiner HL. Evaluation of no evidence of disease activity in a 7-year longitudinal multiple sclerosis cohort. JAMA Neurol. 2015;72:152-158.

92. Hegen H, Bsteh G, Berger T. 'No evidence of disease activity' - is it an appropriate surrogate in multiple sclerosis? Eur J Neurol. 2018;25:1107-e101.

93. Goodin DS, Reder AT, Traboulsee AL, et al. Predictive validity of NEDA in the 16- and 21-year follow-up from the pivotal trial of interferon beta-1b. Mult Scler. 2019;25:837-847.

94. University of California, San Francisco MS-EPIC Team, Cree BA, Gourraud PA, et al. Long-term evolution of multiple sclerosis disability in the treatment era. Ann Neurol. 2016;80:499-510.

95. Río J, Rovira À, Tintoré $\mathrm{M}$, et al. Disability progression markers over 6-12 years in interferon- $\beta$-treated multiple sclerosis patients. Mult Scler. 2018;24:322-330.

96. Prosperini L, Mancinelli CR, Haggiag S, et al. Minimal evidence of disease activity (MEDA) in relapsing-remitting multiple sclerosis. J Neurol Neurosurg Psychiatry. 2020; epublished January 23, 2020.

97. Scalfari A, Neuhaus A, Degenhardt A, et al. The natural history of multiple sclerosis: a geographically based study 10: relapses and long-term disability. Brain. 2010;133(Pt 7):1914-1929.

98. Bermel RA, You X, Foulds P, et al. Predictors of long-term outcome in multiple sclerosis patients treated with interferon $\beta$. Ann Neurol. 2013;73:95-103.

99. Jokubaitis VG, Spelman T, Kalincik T, et al. Predictors of long-term disability accrual in relapse-onset multiple sclerosis. Ann Neurol. 2016;80:89-100.

100. Mowry EM, Pesic M, Grimes B, Deen S, Bacchetti P, Waubant E. Demyelinating events in early multiple sclerosis have inherent severity and recovery. Neurology. 2009;72:602-608.

101. Naldi P, Collimedaglia L, Vecchio D, et al. Predictors of attack severity and duration in multiple sclerosis: a prospective study. Open Neurol J. 2011;5:75-82.

102. Stewart T, Spelman T, Havrdova E, et al. Contribution of different relapse phenotypes to disability in multiple sclerosis. Mult Scler. 2017;23:266-276.

103. Kurtzke JF. Rating neurologic impairment in multiple sclerosis: an expanded disability status scale (EDSS). Neurology. 1983;33: 1444-1452.

104. Kalincik T, Cutter G, Spelman T, et al. Defining reliable disability outcomes in multiple sclerosis. Brain. 2015;138(Pt 11): 3287-3298.

105. Kaufman M, Moyer D, Norton J. The significant change for the Timed 25-foot Walk in the multiple sclerosis functional composite. Mult Scler. 2000;6:286-290.

106. Schwid SR, Goodman AD, McDermott MP, Bever CF, Cook SD. Quantitative functional measures in MS: what is a reliable change? Neurology. 2002;58:1294-1296.

107. Feys P, Lamers I, Francis G, et al. The Nine-Hole Peg Test as a manual dexterity performance measure for multiple sclerosis. Mult Scler. 2017;23:711-720.

108. Hohol MJ, Orav EJ, Weiner HL. Disease steps in multiple sclerosis: a simple approach to evaluate disease progression. Neurology. 1995;45:251-255.

109. Learmonth YC, Motl RW, Sandroff BM, Pula JH, Cadavid D. Validation of patient determined disease steps (PDDS) scale scores in persons with multiple sclerosis. BMC Neurol. 2013;13:37.

110. Norris M, Anderson R, Motl RW, Hayes S, Coote S. Minimum number of days required for a reliable estimate of daily step count and energy expenditure, in people with MS who walk unaided. Gait Posture. 2017;53:201-206.

111. Motl RW, Pilutti LA, Learmonth YC, Goldman MD, Brown T. Clinical importance of steps taken per day among persons with multiple sclerosis. PLoS One. 2013;8:e73247.

112. Lorscheider J, Benkert P, Schädelin S, et al. Disability progression unrelated to relapses in relapsing-remitting multiple sclerosis: insights from the Swiss multiple sclerosis cohort study (abstract 273). Presented at the 35th Congress of the European Committee for Treatment and Research in Multiple Sclerosis, Stockholm, Sweden, October 11-13, 2019.

113. Traboulsee A, Simon JH, Stone L, et al. Revised recommendations of the Consortium of MS Centers Task Force for a standardized MRI protocol and clinical guidelines for the diagnosis and follow-up of multiple sclerosis. AJNR Am J Neuroradiol. 2016;37:394-401.

114. Miller DH, Rudge P, Johnson G, et al. Serial gadolinium enhanced magnetic resonance imaging in multiple sclerosis. Brain. 1988; 111(pt 4):927-939.

115. Bellanger G, Biotti D, Patsoura $S$, et al. What is the relevance of the systematic use of gadolinium during the MRI follow-up of multiple sclerosis patients under natalizumab? Clin Neuroradiol. 2019; epublished May 29, 2019.

116. Tsantes E, Curti E, Puci F, et al. The contribution of enhancing lesions in monitoring multiple sclerosis treatment: is gadolinium always necessary? Abstract EPO3207. Presented at the European Academy of Neurology annual meeting, June 29-July 2, 2019.

117. Zivadinov R, Bergsland N, Hagemeier J, et al. Cumulative gadodiamide administration leads to brain gadolinium deposition in early MS. Neurology. 2019;93:e611-e623.

118. Zecca C, Disanto G, Sormani MP, et al. Relevance of asymptomatic spinal MRI lesions in patients with multiple sclerosis. Mult Scler. 2016;22:782-791.

119. Granella F, Tsantes E, Graziuso S, Bazzurri V, Crisi G, Curti E. Spinal cord lesions are frequently asymptomatic in relapsingremitting multiple sclerosis: a retrospective MRI survey. J Neurol. 2019;266:3031-3037.

120. Casserly C, Seyman EE, Alcaide-Leon P, et al. Spinal cord atrophy in multiple sclerosis: a systematic review and meta-analysis. J Neuroimaging. 2018;28:556-586.

121. Opfer R, Ostwaldt AC, Sormani MP, et al. Estimates of agedependent cutoffs for pathological brain volume loss using SIENA/FSL-a longitudinal brain volumetry study in healthy adults. Neurobiol Aging. 2018;65:1-6.

122. Opfer R, Ostwaldt AC, Walker-Egger C, et al. Within-patient fluctuation of brain volume estimates from short-term repeated MRI measurements using SIENA/FSL. J Neurol. 2018;265: $1158-1165$.

123. Galassi S, Prosperini L, Logoteta A, et al. A lesion topographybased approach to predict the outcomes of patients with multiple sclerosis treated with interferon beta. Mult Scler Relat Disord. 2016;8:99-106.

124. Chard DT, Brex PA, Ciccarelli O, et al. The longitudinal relation between brain lesion load and atrophy in multiple sclerosis: a 14 year follow up study. J Neurol Neurosurg Psychiatry. 2003;74: $1551-1554$

125. Sombekke MH, Wattjes MP, Balk LJ, et al. Spinal cord lesions in patients with clinically isolated syndrome: a powerful tool in diagnosis and prognosis. Neurology. 2013;80:69-75.

126. Brownlee WJ, Altmann DR, Prados F, et al. Early imaging predictors of long-term outcomes in relapse-onset multiple sclerosis. Brain. 2019;142:2276-2287.

127. Planche V, Gibelin M, Cregut D, Pereira B, Clavelou P. Cognitive impairment in a population-based study of patients with multiple sclerosis: differences between late relapsing-remitting, secondary progressive and primary progressive multiple sclerosis. Eur $\mathbf{J}$ Neurol. 2016;23:282-289.

128. Rao SM, Leo GJ, Ellington L, Nauertz T, Bernardin L, Unverzagt F. Cognitive dysfunction in multiple sclerosis. II. Impact on employment and social functioning. Neurology. 1991;41:692-696.

129. Ruet A, Deloire M, Hamel D, Ouallet JC, Petry K, Brochet B. Cognitive impairment, health-related quality of life and vocational status at early stages of multiple sclerosis: a 7-year longitudinal study. J Neurol. 2013;260:776-784. 
130. Morrow SA, Classen S, Monahan M, et al. On-road assessment of fitness-to-drive in persons with MS with cognitive impairment: a prospective study. Mult Scler. 2018;24:1499-1506.

131. Kalb R, Beier M, Benedict RHB, et al. Recommendations for cognitive screening and management in multiple sclerosis care. Mult Scler. 2018;24:1665-1680.

132. Benedict RHB, DeLuca J, Phillips G, et al. Validity of the Symbol Digit Modalities Test as a cognition performance outcome measure for multiple sclerosis. Mult Scler. 2017;23:721-733.

133. Walker LAS, Marino D, Berard JA, Feinstein A, Morrow SA, Cousineau D. Canadian normative data for minimal assessment of cognitive function in multiple sclerosis. Can J Neurol Sci. 2017;44:547-555.

134. Morrow SA, O'Connor PW, Polman CH, et al. Evaluation of the symbol digit modalities test (SDMT) and MS neuropsychological screening questionnaire (MSNQ) in natalizumab-treated MS patients over 48 weeks. Mult Scler. 2010;16:1385-1392.

135. Benedict RH, Morrow S, Rodgers J, et al. Characterizing cognitive function during relapse in multiple sclerosis. Mult Scler. 2014;20:1745-1752.

136. Riepl E, Pfeuffer S, Ruck T, et al. Alemtuzumab improves cognitive processing speed in active multiple sclerosis-a longitudinal observational study. Front Neurol. 2018;8:730.

137. Gudesblatt M, Wissemann $\mathrm{K}$, Zarif $\mathrm{M}$, et al. Improvement in cognitive function as measured by NeuroTrax in patients with relapsing multiple sclerosis treated with natalizumab: a 2-year retrospective analysis. CNS Drugs. 2018;32:1173-1181.

138. Benedict RH, de Seze J, Hauser SL, et al. Impact of ocrelizumab on cognition in patients at increased risk of developing progressive disease (abstract DX67) Presented at the Consortium of Multiple Sclerosis Centers annual meeting, Nashville TN, May 30-June 2, 2018.

139. Zipoli V, Tortorella P, Goretti B, et al. Effect of delayed-release dimethyl fumarate on cognition in Italian patients with relapsing remitting multiple sclerosis: the phase 4 StarTec study (abstract P457). Presented at the 34th Congress of the European Committee for Treatment and Research in Multiple Sclerosis, Berlin, Germany, October 10-12, 2018.

140. Cree BA, Goldman MD, Corboy JR, et al. Effect of fingolimod on functional disability, cognition and quality of life outcomes versus glatiramer acetate in relapsing-remitting multiple sclerosis patients: results from the ASSESS study (abstract EPO1230). Presented at the 5th Congress of the European Academy of Neurology, Oslo, Norway, June 29-July 2, 2019.

141. Sprenger T, Kappos L, Radue EW, et al. Association of brain volume loss and long-term disability outcomes in patients with multiple sclerosis treated with teriflunomide. Mult Scler. 2019; epublished June 14, 2019.

142. Chalmer TA, Kalincik T, Laursen B, Sorensen PS, Magyari M, Members of Danish Multiple Sclerosis Group. Treatment escalation leads to fewer relapses compared with switching to another moderately effective therapy. J Neurol. 2019;266:306-315.

143. Zhovtis Ryerson L, Frohman TC, Foley J, et al. Extended interval dosing of natalizumab in multiple sclerosis. J Neurol Neurosurg Psychiatry. 2016;87:885-889.

144. Scarpazza C, De Rossi N, Tabiadon G, Turrini MV, Gerevini S, Capra R. Four cases of natalizumab-related PML: a less severe course in extended interval dosing? Neurol Sci. 2019;40:2119-2124.

145. Prosperini L, Kinkel RP, Miravalle AA, Iaffaldano P, Fantaccini S. Post-natalizumab disease reactivation in multiple sclerosis: systematic review and meta-analysis. Ther Adv Neurol Disord. 2019;12:1756286419837809.

146. Vollmer B, Honce JM, Sillau S, et al. The impact of very short transition times on switching from natalizumab to fingolimod on imaging and clinical effectiveness outcomes in multiple sclerosis. J Neurol Sci. 2018;390:89-93.

147. Pfeuffer S, Schmidt R, Straeten FA, et al. Efficacy and safety of alemtuzumab versus fingolimod in RRMS after natalizumab cessation. J Neurol. 2019;266:165-173.

148. Fragoso YD, Adoni T, Gomes S, et al. Severe exacerbation of multiple sclerosis following withdrawal of fingolimod. Clin Drug Investig. 2019;39:909-913.
149. Vermersch P, Radue EW, Putzki N, Ritter S, Merschhemke M, Freedman MS. A comparison of multiple sclerosis disease activity after discontinuation of fingolimod and placebo. Mult Scler J Exp Transl Clin. 2017;3:2055217317730096.

150. Schmidt S, Schulten T. Severe rebound after cessation of fingolimod treated with ocrelizumab with coincidental transient aggravation: report of two cases. Ther Adv Neurol Disord. 2019; 12:1756286419846818.

151. Holmøy T, Torkildsen $\varnothing$, Zarnovicky S. Extensive multiple sclerosis reactivation after switching from fingolimod to rituximab. Case Rep Neurol Med. 2018;2018:5190794.

152. Bernard-Valnet R, Pignolet B, Biotti D, et al. Unexpected high multiple sclerosis activity after switching from fingolimod to alemtuzumab. Mult Scler Relat Disord. 2018;25:216-218.

153. Huhn K, Bayas A, Doerck S, et al. Alemtuzumab as rescue therapy in a cohort of 50 relapsing-remitting MS patients with breakthrough disease on fingolimod: a multi-center observational study. J Neurol. 2018;265:1521-1527.

154. Alcalá C, Gascón F, Pérez-Miralles F, Domínguez JA, Gil-Perotín S, Casanova B. Treatment with alemtuzumab or rituximab after fingolimod withdrawal in relapsing-remitting multiple sclerosis is effective and safe. J Neurol. 2019;266:726-734.

155. Cabrera-Maqueda JM, Fuentes-Rumí L, Carreón-Guarnizo E, et al. Lymphocyte subsets recovery time following first alemtuzumab course in multiple sclerosis patients previously treated with fingolimod (abstract P935). Presented at the 34th Congress of the European Committee for Treatment and Research in Multiple Sclerosis, Berlin, Germany, October 10-12, 2018.

156. Mavenclad (cladribine) Product Monograph. EMD Serono, November 29, 2017.

157. Cook S, Leist T, Comi G, et al. Safety of cladribine tablets in the treatment of patients with multiple sclerosis: an integrated analysis. Mult Scler Relat Disord. 2019;29:157-167.

158. Giovannoni G, Soelberg Sorensen P, Cook S, et al. Safety and efficacy of cladribine tablets in patients with relapsingremitting multiple sclerosis: results from the randomized extension trial of the CLARITY study. Mult Scler. 2018;24: 1594-1604

159. Mayer L, Kappos L, Racke MK, et al. Ocrelizumab infusion experience in patients with relapsing and primary progressive multiple sclerosis: results from the phase 3 randomized OPERA I, OPERA II, and ORATORIO studies. Mult Scler Relat Disord. 2019;30:236-243.

160. Manners S. PML reported in patient newly-treated with ocrelizumab, NeuroSens, October 30, 2019. http://neuro-sens.com/ pml-reported-in-patient-newly-treated-with-ocrelizumab/. Accessed 30 October 2019.

161. Ciardi MR, Iannetta M, Zingaropoli MA, et al. Reactivation of hepatitis B virus with immune-escape mutations after ocrelizumab treatment for multiple sclerosis. Open Forum Infect Dis. 2018;6:ofy356.

162. Giovannoni G, Kappos L, Hauser SL, et al. Long-term reduction of relapse rate and confirmed disability progression after 6 years of ocrelizumab treatment in patients with relapsing multiple sclerosis (abstract P1015). Presented at the 35th Congress of the European Committee for Treatment and Research in Multiple Sclerosis, Stockholm, Sweden, September 11-13, 2019.

163. Derfuss T, Weber MS, Hughes R, et al. Serum immunoglobulin levels and risk of serious infections in the pivotal Phase III trials of ocrelizumab in multiple sclerosis and their open-label extensions (abstract 65). Presented at the 35th Congress of the European Committee for Treatment and Research in Multiple Sclerosis, Stockholm, Sweden, September 11-13, 2019.

164. Makatsori M, Kiani-Alikhan S, Manson AL, et al. Hypogammaglobulinaemia after rituximab treatment-incidence and outcomes. QJM. 2014;107:821-828.

165. Baker D, Herrod SS, Alvarez-Gonzalez C, Giovannoni G, Schmierer K. Interpreting lymphocyte reconstitution data from the pivotal phase 3 trials of alemtuzumab. JAMA Neurol. 2017; 74:961-969.

166. Buonomo AR, Zappulo E, Viceconte G, Scotto R, Borgia G, Gentile I. Risk of opportunistic infections in patients treated with 
alemtuzumab for multiple sclerosis. Expert Opin Drug Saf. 2018;17:709-717.

167. Lemtrada (alemtuzumab) Product Monograph. Sanofi Genzyme, January 16, 2019.

168. Montalban X, Arnold DL, Boyko AN, et al. Alemtuzumab maintains efficacy on clinical and MRI disease activity outcomes, including slowing of brain volume loss, over 9 years in RRMS patients: CARE-MS I follow-up (TOPAZ study) (abstract P974). Presented at the 35th Congress of the European Committee for Treatment and Research in Multiple Sclerosis, Stockholm, Sweden, September 11-13, 2019.

169. Schippling S, Bass AD, Boster A, et al. Additional courses of alemtuzumab improved clinical and MRI outcomes in pooled CARE-MS I and II patients with disease activity after three courses: analysis of patients who received $\geq 4$ courses (abstract P628). Presented at the 34th Congress of the European Committee for Treatment and Research in Multiple Sclerosis, Berlin, Germany, October 10-12, 2018.

170. Hyun JW, Kim Y, Kim G, Kim SH, Kim HJ. Severe B cellmediated disease activation despite two cycles of alemtuzumab in a patient with multiple sclerosis. Mult Scler. 2019;25:1942-1945.

171. Wehrum T, Beume LA, Stich O, et al. Activation of disease during therapy with alemtuzumab in 3 patients with multiple sclerosis. Neurology. 2018;90:e601-e605.

172. Massey J, Barnett Y, Curnow J, Sutton I. B cell depletion therapy resulting in sustained remission of severe autoimmune complications following alemtuzumab treatment of multiple sclerosis. Mult Scler Relat Disord. 2019;35:100-103.

173. Muraro PA, Pasquini M, Atkins HL, et al. Long-term outcomes after autologous hematopoietic stem cell transplantation for multiple sclerosis. JAMA Neurol. 2017;74:459-469.

174. Burt RK, Balabanov R, Burman J, et al. Effect of nonmyeloablative hematopoietic stem cell transplantation vs continued diseasemodifying therapy on disease progression in patients with relapsing-remitting multiple sclerosis: a randomized clinical trial. JAMA. 2019;321:165-174.

175. Rush CA, MacLean HJ, Freedman MS. Aggressive multiple sclerosis: proposed definition and treatment algorithm. Nat Rev Neurol. 2015;11:379-389.

176. Fox RJ, Cree BAC, De Sèze J, et al. MS disease activity in RESTORE - a randomized 24-week natalizumab treatment interruption study. Neurology. 2014;82:1491-1498.

177. Havla J, Gerdes LA, Meinl I, et al. De-escalation from natalizumab in multiple sclerosis: recurrence of disease activity despite switching to glatiramer acetate. J Neurol. 2011;258:1665-1669.

178. Cohan SL, Edwards K, Lucas L, et al. Reducing return of disease activity in patients with relapsing multiple sclerosis transitioned from natalizumab to teriflunomide: 12-month interim results of teriflunomide therapy. Mult Scler J Exp Transl Clin. 2019;5: 2055217318824618

179. Cohan SL, Moses H, Calkwood J, et al. Clinical outcomes in patients with relapsing-remitting multiple sclerosis who switch from natalizumab to delayed-release dimethyl fumarate: a multicenter retrospective observational study (STRATEGY). Mult Scler Relat Disord. 2018;22:27-34.

180. Salavisa M, Serrazina F, Ladeira F, Correia AS. Discontinuation of disease-modifying therapy and its clinical impact in MS patients over 60 years (abstract P703). Presented at the 35th Congress of the European Committee for Treatment and Research in Multiple Sclerosis, Stockholm, Sweden, September 11-13, 2019.

181. Birnbaum G. Stopping disease-modifying therapy in nonrelapsing multiple sclerosis: experience from a clinical practice. Int J MS Care. 2017;19:11-14.

182. Schweitzer F, Laurent S, Fink GR, et al. Age and the risks of highefficacy disease modifying drugs in multiple sclerosis. Curr Opin Neurol. 2019;32:305-312.

183. Kalincik T, Malpas C, Sharmin S, et al. Modifiers of the effectiveness of MS immunotherapies (abstract P1421). Presented at the 35th Congress of the European Committee for Treatment and Research in Multiple Sclerosis, Stockholm, Sweden, October 11-13, 2019.

184. Bastian I, Coulter C. National Tuberculosis Advisory Committee (NTAC). Position statement on interferon- $\gamma$ release assays for the detection of latent tuberculosis infection. Commun Dis Intell Q Rep. 2017;41:E322-E336.

185. Kwon YS, Kim YH, Jeon K, et al. Factors that predict negative results of QuantiFERON-TB Gold In-Tube test in patients with culture-confirmed tuberculosis: a multicenter retrospective cohort study. PLoS One. 2015;10:e0129792.

186. Baber U, Bouley A, Egnor E, Sloane JA. Anti-JC virus antibody index changes in rituximab-treated multiple sclerosis patients. J Neurol. 2018;265:2342-2345.

187. Kadish R, Robertson D, Sweeney M. Fatal leukoencephalopathy in a patient with multiple sclerosis following treatment with ocrelizumab. Neurology. 2018;90(15 Supplement): P5.353.

188. Sinnecker T, Othman J, Kühl M, et al. Progressive multifocal leukoencephalopathy in a multiple sclerosis patient diagnosed after switching from natalizumab to fingolimod. Case Rep Neurol Med. 2016;2016:5876798.

189. Grebenciucova E, Berger JR. Immunosenescence: the role of aging in the predisposition to neuro-infectious complications arising from the treatment of multiple sclerosis. Curr Neurol Neurosci Rep. 2017;17:61

190. Kapoor R, Ho PR, Campbell N, et al. Effect of natalizumab on disease progression in secondary progressive multiple sclerosis (ASCEND): a phase 3, randomised, double-blind, placebocontrolled trial with an open-label extension. Lancet Neurol. 2018;17:405-415.

191. Paolillo A, Coles AJ, Molyneux PD, et al. Quantitative MRI in patients with secondary progressive MS treated with monoclonal antibody Campath 1H. Neurology. 1999;53:751-757.

192. Lublin F, Miller DH, Freedman MS, et al. Oral fingolimod in primary progressive multiple sclerosis (INFORMS): a phase 3, randomised, double-blind, placebo-controlled trial. Lancet. 2016;387:1075-1084.

193. Luchetti S, Fransen NL, van Eden CG, Ramaglia V, Mason M, Huitinga I. Progressive multiple sclerosis patients show substantial lesion activity that correlates with clinical disease severity and sex: a retrospective autopsy cohort analysis. Acta Neuropathol. 2018;135:511-528

194. Frischer JM, Weigand SD, Guo Y, et al. Clinical and pathological insights into the dynamic nature of the white matter multiple sclerosis plaque. Ann Neurol. 2015;78:710-721.

195. Mayzent (siponimod) U.S. Product Monograph. Novartis Pharmaceuticals Corp., March 26, 2019.

196. Kappos L, Bar-Or A, Cree BAC, et al. Siponimod versus placebo in secondary progressive multiple sclerosis (EXPAND): a doubleblind, randomised, phase 3 study. Lancet. 2018;391:1263-1273.

197. Mavenclad (cladribine) U.S. Product Monograph. EMD Serono, March 29, 2019.

198. Rice GP, Filippi M, Comi G. Cladribine and progressive MS: clinical and MRI outcomes of a multicenter controlled trial. Cladribine MRI Study Group. Neurology. 2000;54:1145-1155.

199. Beutler E, Sipe JC, Romine JS, Koziol JA, McMillan R, Zyroff J. The treatment of chronic progressive multiple sclerosis with cladribine. Proc Natl Acad Sci USA. 1996;93:1716-1720.

200. Kister I, Spelman T, Alroughani R, et al. Discontinuing diseasemodifying therapy in MS after a prolonged relapse-free period: a propensity score-matched study. J Neurol Neurosurg Psychiatry. 2016;87:1133-1137.

201. Cohen JA, Cutter GR, Fischer JS, et al. Benefit of interferon beta-1a on MSFC progression in secondary progressive MS. Neurology. 2002;59:679-687.

202. Panitch H, Miller A, Paty D, Weinshenker B, North American Study Group on Interferon beta-1b in Secondary Progressive MS. Interferon beta-1b in secondary progressive MS: results from a 3-year controlled study. Neurology. 2004;63:1788-1795.

203. Knox KB, Saini A, Levin MC. The dilemma of when to stop disease-modifying therapy in multiple sclerosis: a narrative review and Canadian regional reimbursement policies. Int J MS Care. 2019. Published online: 22 May 2019. doi:10.7224/15372073.2018-107

204. Ayrignac X, Bilodeau PA, Prat A, et al. Assessing the risk of multiple sclerosis disease-modifying therapies. Expert Rev Neurother. 2019;19:695-706. 
205. Hughes SE, Spelman T, Gray OM, et al. Predictors and dynamics of postpartum relapses in women with multiple sclerosis. Mult Scler. 2014;20:739-746.

206. Portaccio E, Tudisco L, Pastò L, et al. Pregnancy in women with active multiple sclerosis increases the risk of disability progression (abstract P409). Presented at the 35th Congress of the European Committee for Treatment and Research in Multiple Sclerosis, Stockholm, Sweden, October 11-13, 2019.

207. Coyle PK, Oh J, Magyari M, Oreja-Guevara C, Houtchens M. Management strategies for female patients of reproductive potential with multiple sclerosis: an evidence-based review. Mult Scler Relat Disord. 2019;32:54-63.

208. Gilenya (fingolimod) Product Monograph. Novartis Pharmaceuticals Canada, May 2, 2019.

209. Portaccio E, Annovazzi P, Ghezzi A, et al. Pregnancy decisionmaking in women with multiple sclerosis treated with natalizumab: I: Fetal risks. Neurology. 2018;90:e823-e831.

210. Haghikia A, Langer-Gould A, Rellensmann G, et al. Natalizumab use during the third trimester of pregnancy. JAMA Neurol. 2014;71:891-895.

211. Hellwig K, Haghikia A, Rockhoff M, Gold R. Multiple sclerosis and pregnancy: experience from a nationwide database in Germany. Ther Adv Neurol Disord. 2012;5:247-253.

212. Avila-Ornelas J, Avila M, Stosic M, et al. The role of postpartum intravenous corticosteroids in the prevention of relapses in multiple sclerosis. Int J MS Care. 2011;13:91-93.

213. Winkelmann A, Rommer PS, Hecker M, Zettl UK. Intravenous immunoglobulin treatment in multiple sclerosis: a prospective, rater-blinded analysis of relapse rates during pregnancy and the postnatal period. CNS Neurosci Ther. 2019;25:78-85.

214. Almas S, Vance J, Baker T, Hale T. Management of multiple sclerosis in the breastfeeding mother. Mult Scler Int. 2016;2016: 6527458.

215. Polman CH, Reingold SC, Banwell B, et al. Diagnostic criteria for multiple sclerosis: 2010 revisions to the McDonald criteria. Ann Neurol. 2011;69:292-302.

216. Hennes EM, Baumann M, Lechner C, Rostásy K. MOG spectrum disorders and role of MOG-antibodies in clinical practice. Neuropediatrics. 2018;49:3-11.

217. Krysko KM, Graves J, Rensel M, et al. Use of newer diseasemodifying therapies in pediatric multiple sclerosis in the US. Neurology. 2018;91:e1778-e1787.

218. Chitnis T, Arnold DL, Banwell B, et al Trial of fingolimod versus interferon beta-1a in pediatric multiple sclerosis. N Engl J Med. 2018;379:1017-1027.

219. Chitnis T, Ghezzi A, Bajer-Kornek B, Boyko A, Giovannoni G, Pohl D. Pediatric multiple sclerosis: escalation and emerging treatments. Neurology. 2016;87(9 Suppl 2):S103-S109.

220. Huppke B, Ellenberger D, Hummel H, et al. Association of obesity with multiple sclerosis risk and response to first-line disease modifying drugs in children. JAMA Neurol. 2019; epublished July 15, 2019.

221. McKay KA, Manouchehrinia A, Berrigan L, Fisk JD, Olsson T, Hillert J. Long-term cognitive outcomes in patients with pediatriconset vs adult-onset multiple sclerosis. JAMA Neurol. 2019; epublished June 17, 2019. 